\title{
Far-forward neutrinos at the Large Hadron Collider
}

\author{
Weidong Bai, ${ }^{a}$ Milind Diwan, ${ }^{b}$ Maria Vittoria Garzelli, ${ }^{c}$ Yu Seon Jeong ${ }^{d}$ \\ and Mary Hall Reno ${ }^{a}$ \\ ${ }^{a}$ Department of Physics and Astronomy, University of Iowa, \\ Iowa City, IA 52242, U.S.A. \\ ${ }^{b}$ Brookhaven National Laboratory, \\ Upton, NY 11973-5000, U.S.A. \\ ${ }^{c}$ Università degli Studi di Firenze, Dipartimento di Fisica e Astronomia \& INFN, \\ Firenze, Italy \\ 'Theoretical Physics Department, CERN, \\ 1211 Geneva 23, Switzerland \\ E-mail: weidong-bai@uiowa.edu, diwan@bnl.gov, garzelli@mi.infn.it, \\ yuseon.jeong@cern.ch, mary-hall-reno@uiowa.edu
}

ABSTRACT: We present a new calculation of the energy distribution of high-energy neutrinos from the decay of charm and bottom hadrons produced at the Large Hadron Collider (LHC). In the kinematical region of very forward rapidities, heavy-flavor production and decay is a source of tau neutrinos that leads to thousands of charged-current tau neutrino events in a $1 \mathrm{~m}$ long, $1 \mathrm{~m}$ radius lead neutrino detector at a distance of $480 \mathrm{~m}$ from the interaction region. In our computation, next-to-leading order QCD radiative corrections are accounted for in the production cross-sections. Non-perturbative intrinsic- $k_{T}$ effects are approximated by a simple phenomenological model introducing a Gaussian $k_{T}$-smearing of the parton distribution functions, which might also mimic perturbative effects due to multiple initial-state soft-gluon emissions. The transition from partonic to hadronic states is described by phenomenological fragmentation functions. To study the effect of various input parameters, theoretical predictions for $D_{s}^{ \pm}$production are compared with LHCb data on double-differential cross-sections in transverse momentum and rapidity. The uncertainties related to the choice of the input parameter values, ultimately affecting the predictions of the tau neutrino event distributions, are discussed. We consider a $3+1$ neutrino mixing scenario to illustrate the potential for a neutrino experiment to constrain the $3+1$ parameter space using tau neutrinos and antineutrinos. We find large theoretical uncertainties in the predictions of the neutrino fluxes in the far-forward region. Untangling the effects of tau neutrino oscillations into sterile neutrinos and distinguishing a $3+1$ scenario from the standard scenario with three active neutrino flavours, will be challenging due to the large theoretical uncertainties from QCD.

Keywords: Beyond Standard Model, Heavy Quark Physics, Neutrino Physics

ARXiv EPrint: 2002.03012 


\section{Contents}

1 Introduction 1

2 Overview of forward neutrino detection geometry 4

3 Forward heavy-flavor production and decay at the LHC 5

4 Neutrinos from the heavy-flavor hadrons $\quad 12$

$\begin{array}{ll}4.1 \text { Tau neutrinos } & 12\end{array}$

$\begin{array}{lll}4.2 & \text { Muon neutrinos } & 18\end{array}$

$\begin{array}{lll}5 & \text { New physics } & 23\end{array}$

6 Conclusions 28

$\begin{array}{ll}\text { A Decay distributions } & 30\end{array}$

\section{Introduction}

Since the discovery of oscillation properties of neutrinos, their fundamental roles in shaping the universe have become an important line of inquiry [1]. A high-luminosity neutrino program with a flux of neutrinos produced at an energy scale of few $\mathrm{GeV}$ is the focus of the large experimental neutrino physics community working on the Deep Underground Neutrino Experiment (DUNE) [2-4]. With a proton beam energy of 80-120 GeV, pions are produced with the highest multiplicity in proton-nucleon interactions. Thus the beams at DUNE are predominantly muon neutrino (and muon antineutrino) beams, generated by charged pion decays. Future measurements of muon neutrino disappearance and electron neutrino appearance, and of the differences between neutrino and antineutrino rates, will allow the extraction of elements of the Pontecorvo-Maki-Nakagawa-Sakata (PMNS) mixing matrix with better precision than they are currently known. The mixing of tau neutrinos with muon and electron neutrinos will be determined indirectly through disappearance and appearance probabilities since at these energies tau neutrinos are not directly produced in the beam and the tau mass threshold severely suppresses the number of tau neutrino charged-current events at the far detector.

Recently, attention has turned to opportunities of measuring the interactions of highly energetic tau neutrinos produced by $p p$ collisions at the LHC [5-11]. Tau neutrino beams would allow for direct tests of lepton universality and to explore tau neutrino PMNS mixing in the traditional three-neutrino mixing paradigm and in scenarios including additional exotic neutrinos. It was already recognized several decades ago that through the production and prompt decays of the $D_{s}^{ \pm}$and $B$ mesons to taus and tau neutrinos, hadron colliders 
produce large fluxes of tau neutrinos in the forward direction [12-15]. Electron and muon neutrinos and antineutrinos from $D$ and $B$ meson decays will also be produced. Charm and bottom quark production in the Standard Model mostly occurs in quark-antiquark pairs, leading to an approximately equal number of hadrons and antihadrons, ${ }^{1}$ so there will be approximately equal fluxes of neutrinos and antineutrinos from heavy flavor. In our discussion below, we will refer to both particles and antiparticles as neutrinos. As described in 1984 by De Rujula and Ruckl [12], using Feynman scaling arguments, a quark-gluon string model and empirical extrapolations based on collider data available at the time, a few thousand tau neutrino events in future $p p$ and $p \bar{p}$ colliders could be detected with a 2.4 ton detector placed $100 \mathrm{~m}$ distant from the interaction point along the tangent to the accelerator arc. An estimation of the neutrino flux using Pyтнia [17] tuned to Tevatron data and rescaled to a $\sqrt{s}=14 \mathrm{TeV}$ center-of-mass energy gives qualitatively consistent event rates [5].

In the last few years, there is renewed interest in far-forward neutrino production and detection [6-11, 18, 19]. The ForwArd Search ExpeRiment at the LHC (FASER), primarily dedicated to searches for light, extremely weakly interacting particles [6-8], with phase 1 approved and under construction, will be sensitive to tau neutrinos if the detector mass is sufficiently large. The location of the detector is projected to be $480 \mathrm{~m}$ from the ATLAS interaction point along the colliding beam axis. The FASER collaboration uses a pseudorapidity cut $\eta>6.87$ on particle momenta (here, neutrinos) in its second phase to determine if they enter a detector of radius $1.0 \mathrm{~m}$. Other evaluations for this baseline use $\eta>6.7$ [9]. For a half-cylinder, 2-meter long lead detector covering pseudorapidities $\eta>6.7$, Beni et al. [10] used Pythia $8[17,20]$ to find $\sim 8,700$ tau neutrino events for a $3,000 \mathrm{fb}^{-1}$ integrated luminosity at the LHC. Other configurations for detectors, for example, in $\eta$ ranges of 8-9.5 and 7.4-8.2 for XSEN [11] and in the range 7.2-8.7 for SND@LHC [21] are also under consideration. The prototype FASER- $\nu$ has $\eta>8.6[18,19]$. We adopt a minimum pseudorapidity $\eta>6.87$ in this work for definiteness. Our results for $\eta>6.7$ are qualitatively similar.

A source of high-energy tau neutrinos opens the possibility of new tests of the Standard Model that can not be done at DUNE. Measurements of LHC forward tau neutrino interactions can be used for direct tests of lepton flavor universality in charged current interactions with much higher statistics than achieved by Donut [22, 23] and OperA [24, 25]. Both Super-Kamiokande and IceCube have reported signs of tau neutrino appearance in their datasets, but the statistics is still very limited [26, 27]. Measurements of the interaction cross-sections of muon neutrinos from heavy-flavor decays at the LHC with the nucleons/nuclei of the target will help to close the gap between direct neutrino cross-section

\footnotetext{
${ }^{1}$ Differences in the forward region between the total number of hadrons and antihadrons from quarkantiquark pair production arise from the recombination of forward final-state heavy quarks with partons from the initial-state protons non participating to the hard scattering, considering the fact that the parton distribution functions of the up and down quarks in the nucleon differ from those of the antiquarks. Baryon-antibaryon asymmetries, in principle, would show a larger effect than meson-antimeson asymmetries. We show below that the $\Lambda_{c}$ gives a small contribution to neutrino production. The $D_{s}^{ \pm}$has no valence component, and experimental studies by LHCb show a $D_{s}^{+}-D_{s}^{-}$asymmetry below $1 \%$ [16].
} 
measurements for $E_{\nu}<370 \mathrm{GeV}$ [1] and the IceCube Collaboration's determination of the averaged cross-section for neutrino plus antineutrino deep-inelastic scattering with nucleons for $E_{\nu}=6.3-980 \mathrm{TeV}$ [28] (see also, e.g., ref. [29]). LHC forward neutrinos will provide the first opportunity for direct neutrino and antineutrino cross-section measurements for neutrino energies up to $E_{\nu} \lesssim 2 \mathrm{TeV}$.

In this paper, we perform a new evaluation of the $D$ and $B$ meson contributions to the $p p$ differential cross section as a function of tau neutrino and muon neutrino energy for $\eta>6.87$. Differently from previous evaluations which are limited to leading order/leading logarithmic accuracy, our evaluation accounts for the effects of next-to-leading order (NLO) QCD radiative corrections to the heavy-quark hadroproduction [30-32] and neutrino deepinelastic-scattering cross-sections. The effects due to the intrinsic transverse momentum of initial state partons confined in the protons are accounted for by a simple model adding $k_{T}$-smearing effects to the standard collinear parton distribution functions (PDF) as an input for the calculation. The same model might also mimic the effects of the resummation of logarithms related to initial-state soft gluon emissions in an approximate and purely phenomenological way. Furthermore, we include a description of the fragmentation of partons into heavy mesons, relying on widely used phenomenological fragmentation functions.

Our main focus is on tau neutrinos which come predominantly from $D_{s}^{ \pm}$decays. To study the effects of different choices of the values of various parameters entering our computation, we compare our theoretical predictions with the LHCb data on $D_{s}^{ \pm}$production [33] in the rapidity range of $2<y<4.5$. These same parameters give predictions in similar agreement with experimental data for other charm hadron distributions at $\mathrm{LHCb}$.

As discussed below, the effect of the transverse momenta of initial state partons can significantly impact the predictions for forward tau neutrino event rates. In general, the LHCb and other charm data give hints of the need for higher-order effects in the description of single-inclusive open D-hadron production, beyond NLO and the limited logarithmic accuracy of the parton shower implementations and of the analytical resummations of various kinds of logarithms presently available. Power suppressed non-perturbative terms might also play a relevant role, considering the smallness of the charm quark mass, still larger but not too large with respect to $\Lambda_{\mathrm{QCD}}$. At present, it is not clear if the discrepancies between theoretical predictions and experimental data at low transverse momenta can be completely cured within the collinear factorization framework, or if it is necessary to go beyond this scenario. Considering the unavailability of rigorous perturbative and non-perturbative QCD theoretical calculations accurate enough to reproduce the shape of the experimental transverse-momentum distributions, we incorporate initial-state partonic $k_{T}$-smearing effects in a purely phenomenological way in the QCD calculation of $D_{s}^{ \pm}$production for the phase space covered by LHCb and assess their impact on the predicted number of neutrino charged-current interaction events.

The elements of the PMNS mixing matrix are least constrained in the tau sector, compared to the other flavor sectors. We demonstrate how sterile neutrino mass and mixing parameters can begin to be constrained by measuring oscillations of LHC tau neutrinos, and we discuss the challenges to pushing these constraints to sterile neutrino masses of order $\sim 20 \mathrm{eV}$. 
Heavy-flavor decays are not the only sources of forward neutrinos, but they dominate the forward tau-neutrino flux. The contributions from $p p \rightarrow W, Z$ production followed by $W, Z$ leptonic decays are negligible for $\eta \gtrsim 6.5$ [10]. On the other hand, for muon and electron neutrinos, charged pion $\left(\pi^{ \pm}\right)$and kaon $\left(K^{ \pm}\right)$decays to $\nu_{\mu}$ and $K_{L} \rightarrow \nu_{e}$ decays $\left(K_{e 3}^{0}\right)$ are most important. For our evaluation of muon and electron neutrino oscillations, we use parametrizations of light meson distributions based on PYTHIA distributions [34].

We begin in section 2 with an overview of the far-forward geometry used for our discussion here. In section 3, we present our $D$ and $B$ hadron production results. Energy distributions of charged-current interaction events generated in a forward detector by neutrinos from heavy-flavor production and decay are shown in section 4 for both tau neutrinos and muon neutrinos. In our estimation, we also account both for muon neutrinos from the decays of charged pions and kaons and for electron neutrinos from kaon decays. Section 5 shows an application to the study of tau neutrino oscillations, considering a $3+1$ oscillation framework with three active neutrinos and one sterile neutrino. We conclude in section 6 . Appendix A collects formulas for the decay distributions to neutrinos.

\section{Overview of forward neutrino detection geometry}

A forward detector along a line tangent to the LHC beam line necessitates calculations in high-pseudorapidity regimes. A detector with radius of $1.2 \mathrm{~m}$ placed at $480 \mathrm{~m}$ from the LHC interaction point is used for the evaluations in ref. [10]. This corresponds to a neutrino pseudorapidity of $\eta>6.7$ for detection. The FASER2 proposal [8] has $R=1.0 \mathrm{~m}$, corresponding to $\eta>6.87$, which we use for the results shown below. Other smaller detectors like XSEN [11] and SND@LHC [21] are discussed in the recent literature. A prototype FASER- $\nu$ at even higher $\eta,{ }^{2}$ with a $25 \mathrm{~cm} \times 25 \mathrm{~cm}$ cross sectional area and length of $1.35 \mathrm{~m}$ of tungsten interleaved with emulsion detectors, will have a few tens of tau neutrino events with an integrated luminosity of $150 \mathrm{fb}^{-1}$ delivered during Run 3 of the LHC [18]. A detector radius of 1.0 meter, for a comparable target mass, increases the number of events by a factor of $\sim 50$ when scaling by cross-sectional area alone.

The LHC interaction region is a very compact source of tau neutrinos. Most of the tau neutrinos come from $D_{s} \rightarrow \nu_{\tau} \tau$ decay. The $\tau \rightarrow \nu_{\tau} X$ decay is also a prompt process. The characteristic size of the region where tau neutrinos are produced by $D_{s}$ decays is of order $\gamma c \tau_{D_{s}} \simeq E_{D_{s}} / m_{D_{s}} \cdot 150 \mu \mathrm{m}$, so of order of $1.5-15 \mathrm{~cm}$ for $E_{D_{s}}=200 \mathrm{GeV}-2 \mathrm{TeV}$. The tau decay length $c \tau=87.11 \mu \mathrm{m}$, multiplied by the $\gamma$-factor for the same energy range, gives a size of $0.98-9.8 \mathrm{~cm}$. Similarly, $\gamma c \tau_{B^{+}}=E_{B^{+}} / m_{B^{+}} \cdot 496 \mu \mathrm{m}$ produces a size of $1.9-19 \mathrm{~cm}$ for the same energy range. Thus, for tau neutrinos produced along the beam pipe, the longitudinal distance is a few to $20 \mathrm{~cm}$. The transverse size is $17 \mu \mathrm{m}$ [35] from the proton bunch size. The compact source means the assumed detector radius of $1 \mathrm{~m}$ and distance of $480 \mathrm{~m}$ from the interaction point translates to a maximum angle relative to the beam axis for the tau neutrino three-momentum of $\theta_{\max }=2.1 \mathrm{mrad}\left(\eta_{\min }=6.87\right)$. This same constraint applies to the momenta of muon and electron neutrinos from heavy-flavor decays.

\footnotetext{
${ }^{2}$ The FASER- $\nu$ experiment has recently been approved. The FASER- $\nu$ detector will be installed in front of FASER. It is expected to be fully operational and collect data during the LHC Run-III.
} 
While the focus of this paper is on heavy-flavor production of neutrinos in the forward region, consideration of oscillations in a $3+1$ mixing framework also requires an estimate of the number of electron and muon neutrinos from both heavy-flavor decays and light-meson decays. In section 4.2, we make an estimation of the production of electron neutrinos and muon neutrinos from light-meson decays. The light-meson decay lengths are long compared to heavy-meson decay lengths, so the detector and magnets near the interaction point play a role. In ref. [18], an evaluation of the number of $\nu_{\mu}+\bar{\nu}_{\mu}$ events in a detector of $25 \mathrm{~cm}$ $\times 25 \mathrm{~cm}$ cross sectional area finds that most of the events below $1 \mathrm{TeV}$ come from charged pion and kaon decays that occur within $55 \mathrm{~m}$ of the interaction point and stay within the opening of the front quadrupole absorber with inner radius of $17 \mathrm{~mm}$. This corresponds to light-meson momenta lying within $1 \mathrm{mrad}$ from the beam axis. A more detailed discussion of this point appears in section 4.2.

Heavy-meson production at small angles with respect to the beam axis receives dominant contributions from the transverse momentum region below few $\mathrm{GeV}$. For example, for $E_{D_{s}}=1 \mathrm{TeV}$, approximating the neutrino direction by the $D_{s}$ direction means that the $p_{T}$ of the $D_{s}$ meson must be smaller than $2.1 \mathrm{GeV}$, and even smaller for lower energies. Non-perturbative effects related to the intrinsic $k_{T}$ of the partons confined in the initial state nucleons are important at such low transverse momenta. Additionally, perturbative effects related to the appearance of large high-energy logarithms together with those due to initital-state multiple soft-gluon emissions, are potentially relevant in the $p_{T}$ range $[0,15]$ $\mathrm{GeV}$ of the $\mathrm{LHCb}$ data considered in this paper, covering low to intermediate $p_{T}$ values.

In our evaluation of differential cross sections for open charm and bottom production, we include Gaussian $k_{T}$-smearing to better match $\mathrm{LHCb}$ data, using a purely phenomenological approach. In this approach the non-perturbative effects are reabsorbed in the $\left\langle k_{T}\right\rangle$ smearing model, which may also mimic part of the all-order perturbative effects in a rough way. We found that a better description of the LHCb data, given the renormalization and factorization scale choices discussed below, is provided in our approach by a $\left\langle k_{T}\right\rangle$ value higher than the naive estimate due to Fermi motion and even larger than the upper end of the range of typical non-perturbative $\left\langle k_{T}\right\rangle$ values of $\sim 1-2 \mathrm{GeV}$ reported in the literature [36-40]. This gives hints, on the one hand, that non-perturbative physics aspects not yet well understood might be particularly relevant for the process we are studying. On the other hand, it hints that the contribution from the Sudakov resummation of double logarithmic perturbative terms related to the emission of an arbitrarily large number of soft gluons, missing in fixed-order calculations, may be large. Since our focus is on $\nu_{\tau}+\bar{\nu}_{\tau}$ production, we use the $\mathrm{LHCb}$ data for $D_{s}$ production at $\sqrt{s}=13 \mathrm{TeV}$ for rapidities in the range of 2.0-4.5 [33].

\section{Forward heavy-flavor production and decay at the LHC}

We evaluate the single-particle inclusive heavy-flavor energy and angular distributions. Our approach relies on perturbation theory (pQCD) in the collinear factorization framework. In particular, we include NLO QCD corrections to the heavy-quark production cross sections [30-32]. 
As noted above, at very high rapidities, the effect of relatively small transverse momenta of the initial state partons can affect the acceptance of neutrino events. In shower Monte Carlo event generators like PYTHIA, the transverse momentum distribution of the produced heavy quarks is affected, on the one hand, by the effects of multiple soft and collinear gluon emissions, accounted for with a limited logarithmic accuracy by the introduction of Sudakov form factors resumming the relative main logarithmic contributions, and, on the other hand, by the inclusion of a small intrinsic transverse momentum, related to the confinement of partons in finite-size nucleons and the uncertainty principle. An alternative to the collinear factorization approach is to use unintegrated parton distribution functions that have a transverse momentum $k_{T}$ dependence in addition to the usual longitudinal momentum fraction $x$ dependence [41, 42]. In both collinear and $k_{T}$ factorization, further higher-order effects can also modify the transverse momentum distribution of heavy-flavor hadroproduction in the low $p_{T}$ region. In particular, the effect of the resummation of high-energy logarithms, not yet implemented into a publicly available code, and the joint resummation of these and other logarithms could also play a relevant role, which deserves future dedicated investigations, but is beyond the scope of this paper.

In the present absence of calculations capable of fully reproducing the experimental shape of the transverse momentum distributions of charm mesons at small transverse momenta at the LHC, our approach here is phenomenological and uses Gaussian smearing of the outgoing charm quark. With the NLO QCD calculation of $g_{\mathrm{NLO}}\left(q_{T}, y\right)=$ $d^{2} \sigma(\mathrm{NLO}) / d q_{T} d y$ for the quark, we evaluate the Gaussian smeared one-particle inclusive charm-quark distribution $g\left(p_{T}, y\right)=d^{2} \sigma / d p_{T} d y$ according to

$$
g\left(p_{T}, y\right)=\int d^{2} \vec{k}_{T} f\left(\vec{k}_{T},\left\langle k_{T}^{2}\right\rangle\right) g_{\mathrm{NLO}}\left(q_{T}=\left|\vec{p}_{T}-\vec{k}_{T}\right|, y\right),
$$

where $f\left(\vec{k}_{T},\left\langle k_{T}^{2}\right\rangle\right)$ is a two-dimensional Gaussian function normalized to unity,

$$
\int d^{2} \vec{k}_{T} f\left(\vec{k}_{T},\left\langle k_{T}^{2}\right\rangle\right)=\int d^{2} \vec{k}_{T} \frac{1}{\pi\left\langle k_{T}^{2}\right\rangle} \exp \left[-k_{T}^{2} /\left\langle k_{T}^{2}\right\rangle\right]=1 .
$$

The smearing generates a shift of the outgoing heavy-quark momentum vector $\vec{q}_{T}$ by $\vec{k}_{T}$ after the hard scattering, before fragmentation. The single Gaussian $f\left(\vec{k}_{T},\left\langle k_{T}^{2}\right\rangle\right)$ is equivalent to starting with two Gaussian functions, one for each incoming parton $\left(k_{i T}\right)$, making a change of variables to $\vec{k}_{T}=\left(\vec{k}_{1 T}+\vec{k}_{2 T}\right) / 2$ for one of the integrals, and integrating over the other parton's $k_{T}$,

$$
d^{2} \vec{k}_{1 T} f\left(\vec{k}_{1 T}, 2\left\langle k_{T}^{2}\right\rangle\right) d^{2} \vec{k}_{2 T} f\left(\vec{k}_{2 T}, 2\left\langle k_{T}^{2}\right\rangle\right) \rightarrow d^{2} \vec{k}_{T} f\left(\vec{k}_{T},\left\langle k_{T}^{2}\right\rangle\right) .
$$

This definition of the effective $\vec{k}_{T}$ variable distributes the transverse momentum smearing equally to the one-particle transverse momentum and to the recoil hadrons [36]. The quantity $\left\langle k_{T}^{2}\right\rangle$ is related to the average magnitude of $\vec{k}_{T},\left\langle k_{T}\right\rangle$, by

$$
\left\langle k_{T}\right\rangle^{2}=\left\langle k_{T}^{2}\right\rangle \pi / 4 \text {. }
$$

As we discuss below, we set the $\left\langle k_{T}^{2}\right\rangle$ value based on comparisons with double-differential distributions in rapidity and transverse momentum for forward $D_{s}$ production measured by $\mathrm{LHCb}$ at $\sqrt{s}=13 \mathrm{TeV}[33]$. 
Charm quark fragmentation to mesons is accounted for by using fragmentation functions of the Peterson form [43]. For $c \rightarrow D^{0}, D^{+}$and $D_{s}^{+}$, we take fragmentation fractions $0.6086,0.2404$ and 0.0802 , respectively [44]. The parameter $\epsilon$ in the Peterson fragmentation function that describes the hardness of the meson spectrum relative to the heavy quark is taken to be $\epsilon=0.028,0.039$ and 0.008 for $D^{0}, D^{+}$and $D_{s}^{+}$, respectively, and $\epsilon=0.003$ for $B$ production [45]. The fragmentation fraction for $B$ 's is $b \rightarrow B^{+}=b \rightarrow B^{0}=0.362$ from ref. [46].

We use the NLO nCTEQ15 parton distribution function (PDF) grids [47], available for free proton and nuclear targets, for our evaluations here. We use their best fit set for free proton targets in our evaluation of charm production in $p p$ collisions. As noted below, we use the NLO nCTEQ15 nuclear PDF set for lead targets in our neutrino cross section calculation. We take a charm pole mass value $m_{c}=1.3 \mathrm{GeV}$ consistent with the choice of PDFs. A $b$-quark pole mass of $4.5 \mathrm{GeV}$ is used here, also consistent with the PDFs. We use renormalization and factorization scales $\left(\mu_{R}, \mu_{F}\right)$ which are factors $\left(N_{R}, N_{F}\right)$ of the transverse mass $m_{T}=\sqrt{m_{Q}^{2}+p_{T}^{2}}$, where $p_{T}$ is the magnitude of the transverse momentum of the heavy quark $Q=c, b$. Conventionally, renormalization and factorization scales are chosen in a range with factors $N_{R}, N_{F}=0.5-2$ in $\left(\mu_{R}, \mu_{F}\right)=\left(N_{R}, N_{F}\right) m_{T}$, with $N_{R}=N_{F}=1$ set as conventional scale factors [48]. The values $N_{R}=1.0$ and $N_{F}=1.5$ lie in the standard scale factor uncertainty range and are used as our default parameters as discussed below, but we also show predictions obtained with the standard conventional choice $N_{R}=N_{F}=1.0$ multiplying $m_{T}$ for $\left(\mu_{R}, \mu_{F}\right)$ used in most, (see, e.g., ref. [48]), though not all (see, e.g., refs. $[49,50]$ ), of the literature on heavy-flavor production. In particular, in the following we will discuss predictions obtained with the following configurations:

- $N_{R}=1, N_{F}=1$ (conventional central scale choice), with $\left\langle k_{T}\right\rangle=0.7 \mathrm{GeV}$,

- $N_{R}=1, N_{F}=1.5$, (alternative central scale choice, used as default in this paper, as better motivated in the following), with $\left\langle k_{T}\right\rangle=0.7 \mathrm{GeV},\left\langle k_{T}\right\rangle=0 \mathrm{GeV}$ and $\left\langle k_{T}\right\rangle=2.2 \mathrm{GeV}$.

The scale input, the PDFs, the fragmentation functions and the non-perturbative transverse momenta all influence the predicted heavy-flavor energy and rapidity distributions. Since our focus is on tau neutrino production, $\mathrm{LHCb}$ data on forward $D_{s}$ production [33] are used to set $\left\langle k_{T}\right\rangle$ for selected $\left(\mu_{R}, \mu_{F}\right)$ combinations, after fixing the PDF and fragmentation function details. The $D_{s}$ data cover the range $0<p_{T}<14 \mathrm{GeV}^{3}$ and $2.0<y<4.5$. While varying the input parameters, a $\chi^{2}$ is computed, which combines the double-differential predictions, the binned data for 71 data points and the experimental uncertainties. As additional constraint, the integrated cross section evaluated with our Monte Carlo integration program is also required to be within the experimental error bars of the measured cross section for the same kinematic region. Fitting the LHCb data for $D_{s}$ production with $p_{T}<14 \mathrm{GeV}$, by minimizing the $\chi^{2}$ with $\mu_{R}=m_{T}, \mu_{F}=1.5 m_{T}$ and varying $\left\langle k_{T}\right\rangle$, leads to $\left\langle k_{T}\right\rangle=2.2 \pm 0.7 \mathrm{GeV}\left(\left\langle k_{T}^{2}\right\rangle=6.2 \mathrm{GeV}^{2}\right)$. This represents a

\footnotetext{
${ }^{3}$ The LHCb measurements reported in ref. [33] extend to a $p_{T}$ value of $15 \mathrm{GeV}$ for $D^{ \pm}, D^{0}$, and $\bar{D}^{0}$, while, for the less abundant $D_{s}^{ \pm}$, the experimental results in the largest $p_{T}$ bin are not reported.
} 

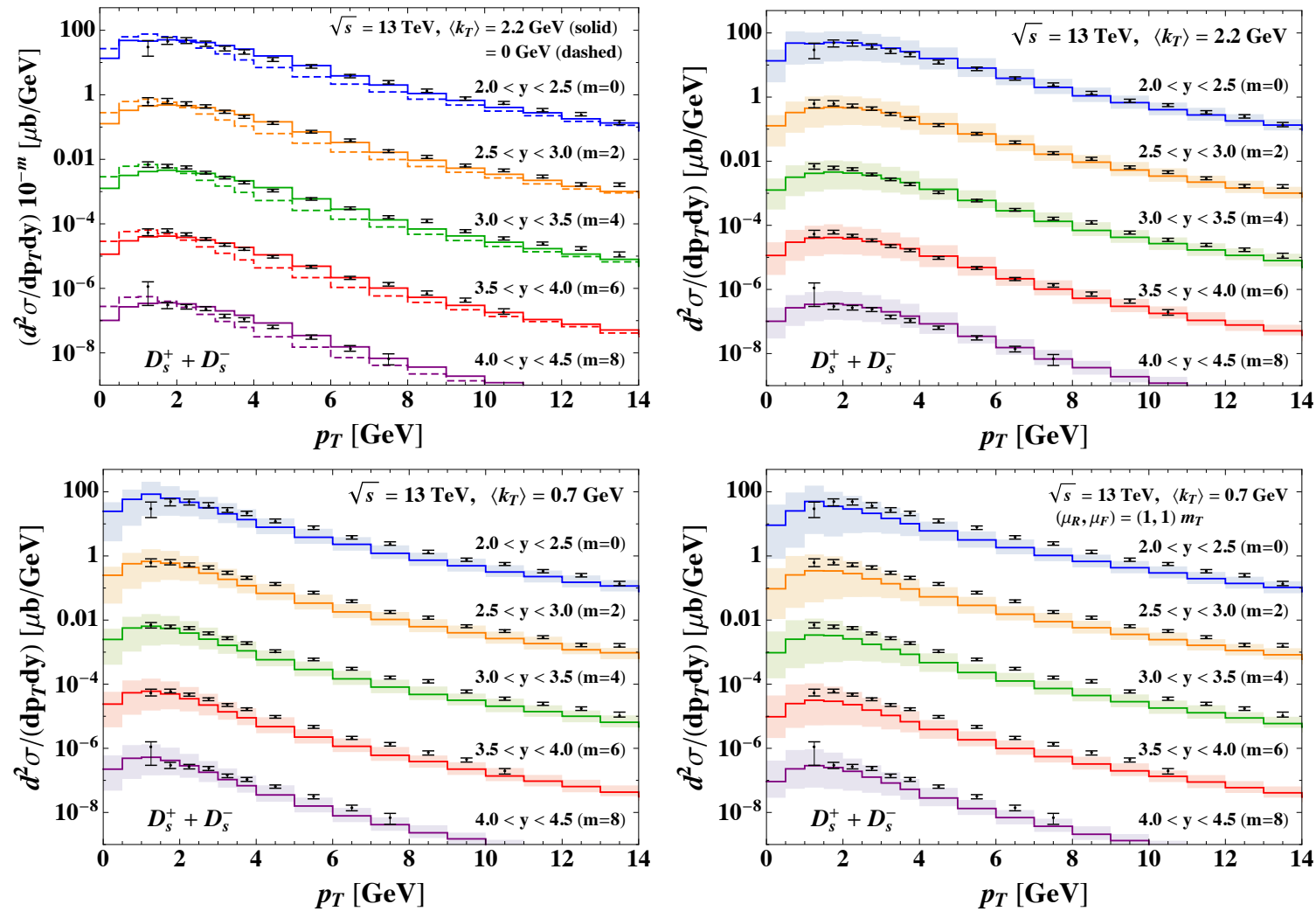

Figure 1. Comparison between LHCb experimental data on double-differential distributions in the meson $p_{T}$ and $y$ for $D_{s}^{ \pm}$production in $p p$ collisions [33] and our QCD predictions. Data (and predictions) for different $\Delta y$ bins are shifted by $10^{-m}$ where values of $m=0,2,4,6$ and 8 . The upper left panel refers to the case $N_{R}=1.0$ and $N_{F}=1.5$, where $\left(\mu_{R}, \mu_{F}\right)=\left(N_{R}, N_{F}\right) m_{T}$, both fixing $\left\langle k_{T}\right\rangle=2.2 \mathrm{GeV}$ and in the collinear approximation $\left(\left\langle k_{T}\right\rangle=0 \mathrm{GeV}\right)$. The upper right and lower left panels refer to the same scale configuration with the shaded band showing the uncertainty built by seven-point scale variation in the range $N_{R, F}=0.5-2$ for $\left\langle k_{T}\right\rangle=2.2 \mathrm{GeV}$ (best fit to data) and $\left\langle k_{T}\right\rangle=0.7 \mathrm{GeV}$ (our default value), respectively. The lower right panel shows the same as lower left, but with $\left(N_{R}, N_{F}\right)=(1,1)$ (conventional scale choice).

reasonably good fit, with $\chi^{2} / \mathrm{DOF}=2.8$ and the cross section within $10 \%$ of the experimentally measured cross section in this kinematic region. With this parameter choice, the theoretical total cross section for $\sigma_{c \bar{c}}$ is within $2 \%$ of the central value of the LHCb estimate reported in ref. [33].

The predictions with $\mu_{R}=m_{T}, \mu_{F}=1.5 m_{T}$ and $\left\langle k_{T}\right\rangle=2.2 \mathrm{GeV}$, together with those for a $\left\langle k_{T}\right\rangle=0$ evaluation using the sames scales and the LHCb data [33], are shown in the upper left panel of figure 1 with the solid and dashed histograms, respectively. From top to bottom, the panel shows data and theoretical evaluations in five different rapidity bins of $\Delta y=0.5$ width for $2.0<y<4.5$ and normalization shifted by $10^{-m}$ where $m=0,2,4,6$, 8. The upper right panel of figure 1 shows, for $\left\langle k_{T}\right\rangle=2.2 \mathrm{GeV}$, the scale uncertainty band obtained as an envelope of seven combinations of scales between factors of 0.5 and 2.0 of the central scales $\left(\mu_{R}, \mu_{F}\right)=(1.0,1.5) m_{T}$, namely with the combinations of $\left(N_{R}, N_{F}\right)$ equal to factors of $m_{T}$ of $(0.5,0.75),(2,3),(1.0,0.75),(0.5,1.5),(1,3),(2.0,1.5)$ and $(1.0,1.5)$ [48]. 
The lower left panel of figure 1 shows the central results and uncertainty band for the same scale choices, now with $\left\langle k_{T}\right\rangle=0.7 \mathrm{GeV}$, the transverse momentum smearing that approximates the Powheg + Pythia results (see figure 2). Finally, the lower right panel shows results with $\left\langle k_{T}\right\rangle=0.7 \mathrm{GeV}$ and the conventional central choice of QCD scales, namely, $N_{R, F}=1.0$.

The uncertainty bands in figure 1 are consistent across the rapidity bins. We expect that the large scale uncertainties here dominate the uncertainties at even higher rapidity. PDF uncertainties are typically smaller than the scale uncertainties for forward charm production at these energies [50]. Results for the $p_{T}$ and $y$ distributions, in similar agreement with the LHCb data, are obtained for $D^{ \pm}$and $D^{0}, \bar{D}^{0}$ production, used below as sources of $\nu_{\mu}+\bar{\nu}_{\mu}$ and $\nu_{e}+\bar{\nu}_{e}$.

In general varying $\left(N_{F}, N_{R}\right)$ changes both the rate and the shape of the distributions. The shape is particularly sensitive to $N_{F}$, whereas the normalization is particularly sensitive to $N_{R}$. At fixed $N_{F}$, the larger $N_{R}$ gives a smaller cross section. The variation of the shape with $N_{F}$ depends on the $\sqrt{s}$. At LHC energies, the $p_{T}$ distribution is steeper for larger $N_{F}$ than for smaller $N_{F}$.

Here, $B$-meson production follows from the same heavy-quark dynamics, with $c \bar{c}$ replaced by $b \bar{b}$, so it is generally expected that the renormalization and factorization scaling factors are the same for both heavy-quark flavors. To the extent that $\left\langle k_{T}\right\rangle$ describes the intrinsic parton transverse momentum in the initial state protons, this parameter also is process independent for $p p \rightarrow Q \bar{Q} X$ for $Q=c, b$. For the same central scale and $\left\langle k_{T}\right\rangle$ choices, the $p_{T}$ distribution of $B^{ \pm}$mesons at $\sqrt{s}=13 \mathrm{TeV}$ for $y=2.0-4.5$ lies $\sim 10 \%$ below the LHCb data [51]. The $B$-decay contribution to the total number of tau neutrino events amounts to less than $10 \%$, as shown in the following, so to approximate bottom production, we use the same scale and $\left\langle k_{T}\right\rangle$ choices as for charm production, with the replacement $m_{c} \rightarrow m_{b}$. Refinements in the parameters used for the description of the $B$-mesons will not change the conclusions of this paper.

Alternatively, one could also consider varying $N_{F}$ and $\left\langle k_{T}\right\rangle$, with $N_{R}$ fixed, to find the two-parameter combination which provides the best fit to the $\mathrm{LHCb} 13 \mathrm{TeV} D_{s}$ data on double-differential cross sections in $p_{T}$ and $y$ at $\sqrt{s}=13 \mathrm{TeV}$. We find that $N_{F}=1.44$ and $\left\langle k_{T}\right\rangle=2.23 \mathrm{GeV}$ is the best fit in that case when $N_{R}=1$, with $\chi^{2} / \mathrm{DOF}=2.68$ and with corresponding predicted $\sigma_{c \bar{c}}$ for $1 \mathrm{GeV}<p_{T}<8 \mathrm{GeV}$ and $2.0<y<4.5$ amounting to $87 \%$ of the central value of the experimental result by the LHCb collaboration, which they extrapolate from $D_{s}$ data. The $3 \sigma$ allowed interval of $\left\langle k_{T}\right\rangle\left(\left\langle k_{T}^{2}\right\rangle\right)$ values turns out to be an ellipse that spans the range $2.02-2.44 \mathrm{GeV}\left(5.20-7.58 \mathrm{GeV}^{2}\right)$ for $N_{F}=1.26-1.62$, favoring a lower $N_{F}$ and higher $\left\langle k_{T}\right\rangle$ (and vice versa) for excursions from the best fit when $N_{R}=1.0$. An examination of the differences between the full charm meson production data from $\mathrm{LHCb}$, their total charm-anticharm pair production cross sections, and theoretical predictions varying simultaneously the three parameters $N_{F}, N_{R}$ and $\left\langle k_{T}\right\rangle$, would be interesting to better understand the roles of $\mu_{R}, \mu_{F}$ and $\left\langle k_{T}\right\rangle$ in theoretical predictions of charm production [52]. ${ }^{4}$ Since our focus here is on $\nu_{\tau}+\bar{\nu}_{\tau}$ production, in the following

\footnotetext{
${ }^{4}$ The parameter $\left\langle k_{T}\right\rangle$ is also tied to the choice of fragmentation function since both influence the heavymeson rapidity and transverse momentum in the collider frame [52].
} 

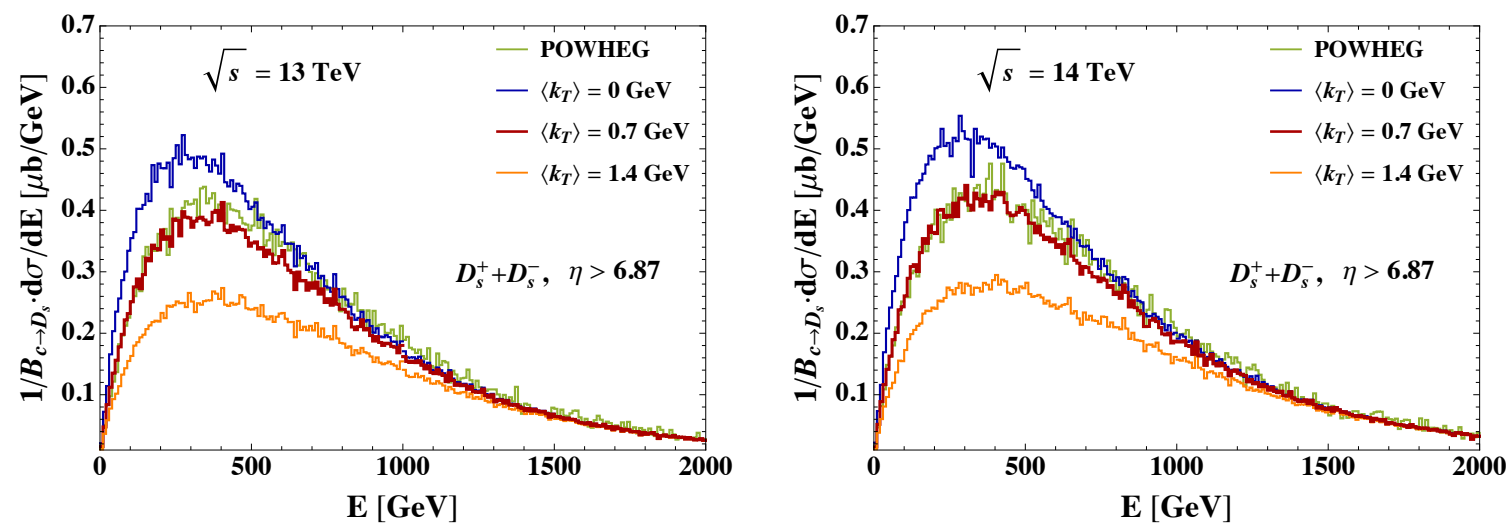

Figure 2. Differential distributions of the energy $E$ of the $D_{s}$ produced in $p p$ collision at $\sqrt{s}=13$ (left) and 14 (right) $\mathrm{TeV}$, for $D_{s}^{ \pm}$pseudorapidity $\eta>6.87$. Predictions obtained within our framework using $\left(\mu_{R}, \mu_{F}\right)=(1.0,1.5) m_{T}$ and $\left\langle k_{T}\right\rangle=0,0.7,1.4 \mathrm{GeV}$, are compared to those of a computation based on NLO hard-scattering matrix-elements matched to parton shower followed by hadronization, according to the PowhEG + PYTHIA framework. See text for more detail.

sections we use $N_{F}=1.5, N_{R}=1.0$ and $\left\langle k_{T}\right\rangle=2.2 \mathrm{GeV}$ as representatives values of the range of parameter choices that lead to a reasonable description of the experimental $\mathrm{LHCb}$ data, together with more widely adopted choices of the same input parameters. As we will see, $\left\langle k_{T}\right\rangle=2.2 \mathrm{GeV}$ leads to a suppression, relative to predictions with smaller $\left\langle k_{T}\right\rangle$, of neutrino production in the forward region.

The large value of $\left\langle k_{T}\right\rangle=2.2 \mathrm{GeV}$ is difficult to reconcile with theoretical expectations for the strong interaction effects that we approximately model with the Gaussian factor. Large $\left\langle k_{T}\right\rangle$ values have been used in some analyses [36, 38, 39]. For example, the NLO evaluation of direct photon production in $p \bar{p}$ collisions at the Tevatron, without resummation effects but including $k_{T}$-smearing with a Gaussian function, shows good agreement with CDF and D0 data when $\left\langle k_{T}\right\rangle=2.5 \mathrm{GeV}\left(\left\langle k_{T}^{2}\right\rangle=8.0 \mathrm{GeV}^{2}\right)$ for the photon [36]. A smaller value of $\left\langle k_{T}^{2}\right\rangle=1 \mathrm{GeV}^{2}$ for the gluon PDF in the unpolarized proton is used to describe polarized proton-unpolarized proton scattering to $J / \psi X$ and $D X$ in ref. [53]. On the other hand, in an analysis of di- $J / \psi$ production at LHCb [54], the unpolarized transverse momentum gluon distribution, factorized in terms of the usual collinear PDF and a Gaussian as in eq. (3.2), yields $\left\langle k_{T}^{2}\right\rangle=3.3 \pm 0.8 \mathrm{GeV}^{2}$ [55]. Non-perturbative Sudakov factors are introduced on top of resummation procedures to describe the transverse momentum distributions of Drell Yan and $W, Z$ production. For example, non-perturbative parameters in impact parameter space that translate to values of $\left\langle k_{T}^{2}\right\rangle$ in the range of $\sim 1-2 \mathrm{GeV}^{2}$ are obtained in ref. [56] to augment the corrections from resummations to better fit the experimental data. One interpretation of the need for a large value of $\left\langle k_{T}\right\rangle$ is that it compensates for missing higher-order perturbative QCD corrections.

The effect of a more conservative value of $\left\langle k_{T}\right\rangle=0.7 \mathrm{GeV}\left(\left\langle k_{T}^{2}\right\rangle=0.6 \mathrm{GeV}^{2}\right)$ is shown in the lower left panel of figure 1 along with the scale uncertainty band, again with our default central scale choices $\left(\mu_{R}, \mu_{F}\right)=(1.0,1.5) m_{T}$. Figure 2 shows predictions for energy distributions for $D_{s}$ production in the very forward region, with $\eta>6.87$, using different ap- 
proaches, at two different center-of-mass energies. Predictions of a computation relying on NLO QCD matrix-elements matched to the parton shower and hadronization algorithms implemented in PythiA, with matching performed according to the PowheG $[57,58]$ method, are compared to those obtained by combining NLO QCD results with the Gaussian transverse momentum smearing model using $\left\langle k_{T}\right\rangle=0,0.7$ and $1.4 \mathrm{GeV}$, followed by fragmentation. All the distributions shown use the same default central scales and charm quark mass. In the NLO + shower Monte Carlo computation, the parameters related to fragmentation and intrinsic transverse momenta are tuned to pre-existing experimental data. The parton shower algorithm accounts for the effect of multiple soft and collinear partonic emissions on top of the hard-scattering process, affecting the kinematics and the dynamics of the event, inducing modifications of the distributions at the fixed-order level. The energy distributions of the Powheg + Pythia computation for $D_{s}$ production in the forward region agree well with our Gaussian smeared NLO predictions when $\left\langle k_{T}\right\rangle=0.7 \mathrm{GeV}$, as shown in both panels of figure 2 . The lower left panel of figure 1 shows that the lower value of $\left\langle k_{T}\right\rangle$ agrees less well with the data at higher transverse momentum but agrees well in case of lower $p_{T}$ of the meson, the kinematic region that dominates in the total cross section and in the production of a forward neutrino beam.

As already mentioned, it is more conventional to use scales equal to $\left(\mu_{R}, \mu_{F}\right)=$ $(1.0,1.0) m_{T}$. We show in the lower right panel of figure 1 the double differential distributions $d^{2} \sigma / d p_{T} d y$ for $D_{s}$ production in different rapidity bins, rescaled as above, obtained using as input of our computation these scales and the same value of $\left\langle k_{T}\right\rangle=0.7 \mathrm{GeV}$ as in the lower left panel. The conventional scale combination $\left(\mu_{R}, \mu_{F}\right)=(1.0,1.0) m_{T}$ typically gives rise to predicted cross sections lower than the LHCb data, at least considering the present limited accuracy of the theoretical calculations, but the data are still included within the large theoretical scale uncertainty bands.

To allow for comparisons with what could be considered more conventional $\left\langle k_{T}\right\rangle$, we show results for both $\left\langle k_{T}\right\rangle=0.7 \mathrm{GeV}$, a value consistent with a fully non-perturbative interpretation of $\left\langle k_{T}\right\rangle$ effects that most closely follows the PowHEG + PYTHIA result, and for $\left\langle k_{T}\right\rangle=2.2 \mathrm{GeV}$, the value corresponding to our best fit of LHCb experimental data. We also show some results with $\left\langle k_{T}\right\rangle=0-1.4 \mathrm{GeV}$. Also for comparisons with other QCD evaluations of heavy-flavor production, we show results for both central scales $\left(\mu_{R}, \mu_{F}\right)=(1.0,1.5) m_{T}$ and for conventional central scales $\left(\mu_{R}, \mu_{F}\right)=(1.0,1.0) m_{T}$, with their respective uncertainty bands that cover the seven point scale variation of $0.5-2$ times the central scale. With an interpretation of $\left\langle k_{T}\right\rangle=2.2 \mathrm{GeV}$, rather than with a value $\sim m_{p}$, as compensating for missing higher order QCD corrections, the seven point scale variations encompass $\left\langle k_{T}\right\rangle$ uncertainties, at least to a degree.

Finally, we illustrate the effect of fragmentation in figure 3. In the left panel, the charm and $D_{s}$ energy distributions are shown for $\eta>4.5$ at $\sqrt{s}=14 \mathrm{TeV}$, while on the right, the energy distribution is shown when $\eta>6.87$. For both panels, the fragmentation fraction for $c \rightarrow D_{s}$ is set to unity to allow a direct comparison of the charm and meson distributions. The right panel of figure 3 shows that the impact of the fragmentation function extends to very high energy in the very forward region. 

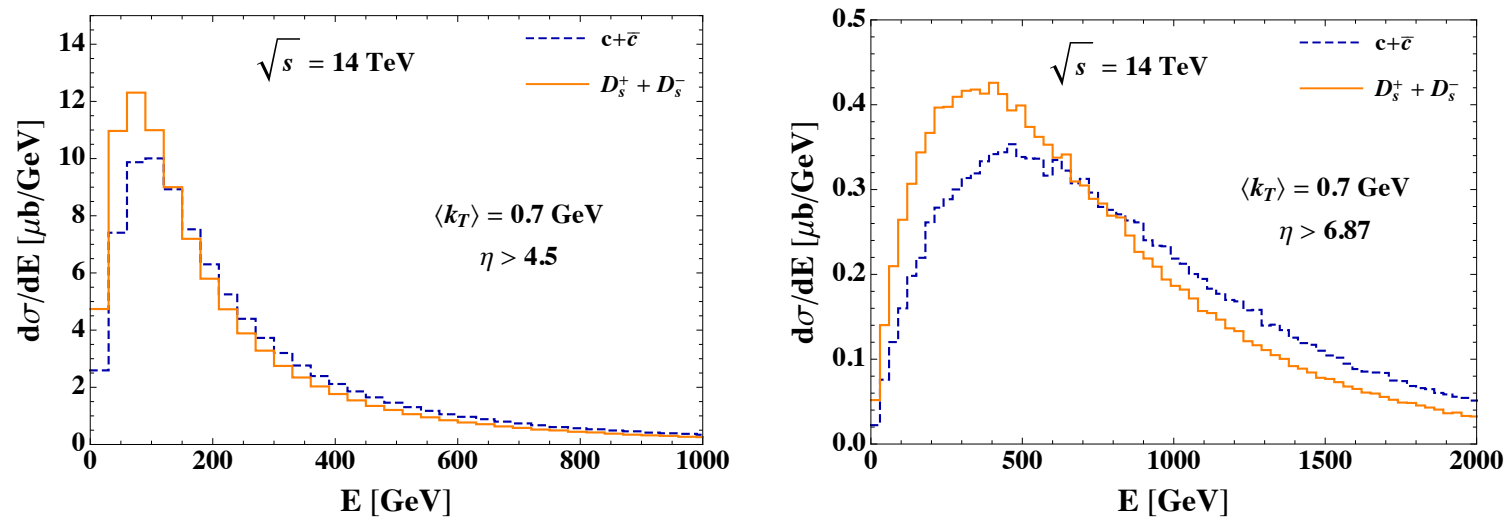

Figure 3. The effect of fragmentation of charm quark to $D_{s}$ which shifts the energy distribution to lower energy for both $\eta>4.5$ (left) and $\eta>6.87$ (right). Shown are the differential cross sections of charm quarks and $D_{s}$ mesons produced in $p p$ collision at $\sqrt{s}=14 \mathrm{TeV}$ for $\left(\mu_{R}, \mu_{F}\right)=(1.0,1.5) m_{T}$ and $\left\langle k_{T}\right\rangle=0.7 \mathrm{GeV}$, as a function of the energy $E$. Note that the $D_{s}$ fragmentation fraction has been set to unity to demonstrate this effect clearly.

\section{Neutrinos from the heavy-flavor hadrons}

\subsection{Tau neutrinos}

Tau neutrinos and antineutrinos arise primarily from the prompt decays of $D_{s}$ mesons into $\tau+\nu_{\tau}$, where $B\left(D_{s} \rightarrow \tau \nu_{\tau}\right)=(5.48 \pm 0.23) \times 10^{-2}$ [45]. The decay of the $\tau$ itself is also prompt and produces a tau neutrino, which is the dominant source of high-energy tau neutrinos in the forward region since the tau carries most of the energy of the $D_{s}$, given $m_{D_{s}}=1.97 \mathrm{GeV}$ and $m_{\tau}=1.78 \mathrm{GeV}$. The energy distribution of the $\nu_{\tau}$ that comes directly from the $D_{s}$ leptonic decay (called the "direct" neutrino) is straightforward to calculate from the isotropic decay of the $D_{s}$ in its rest frame, followed by a boost to the collider frame where the $D_{s}$ has a four-momentum $p_{D}$. Keeping the polarization of the tau [59], the energy distribution of the $\nu_{\tau}$ from the tau decay (here called the "chain" decay neutrino) can also be obtained. The latter distribution, after integrating over angles relative to the tau momentum direction, is discussed in refs. [60, 61] in the context of the atmospheric tau neutrino flux. Here, we use the full energy and angular distribution in the collider frame to apply the requirement that the neutrino pseudorapidity fulfills the $\eta>6.87$ constraint. Details of the tau neutrino energy and angular distribution from $D_{s} \rightarrow \tau \rightarrow \nu_{\tau}$ appear in ref. [62]. The distributions of tau neutrinos and antineutrinos from the $D_{s}$ decays are identical because of the zero-spin of the meson (direct neutrinos) and the compensation of particle/antiparticle and left-handed/right-handed effects in the chain decays of the tau [59].

$B$-meson production and decay also contributes to the number of tau neutrinos, but at a level of less than $10 \%$ of the contribution from $D_{s}$ production and decay. The branching fractions to taus from charged and neutral $B$ 's are $B\left(B^{+} \rightarrow \bar{D}^{0} \tau^{+} \nu_{\tau}\right)=(7.7 \pm 2.5) \times 10^{-3}$, $B\left(B^{+} \rightarrow \bar{D}^{*}(2007)^{0} \tau^{+} \nu_{\tau}\right)=(1.88 \pm 0.20) \times 10^{-2}, B\left(B^{0} \rightarrow D^{-} \tau^{+} \nu_{\tau}=(1.08 \pm 0.23) \times 10^{-2}\right.$, and $B\left(B^{0} \rightarrow D^{*}(2010)^{-} \tau^{+} \nu_{\tau}\right)=(1.57 \pm 0.10) \times 10^{-2}$ [45]. We use the central values of 

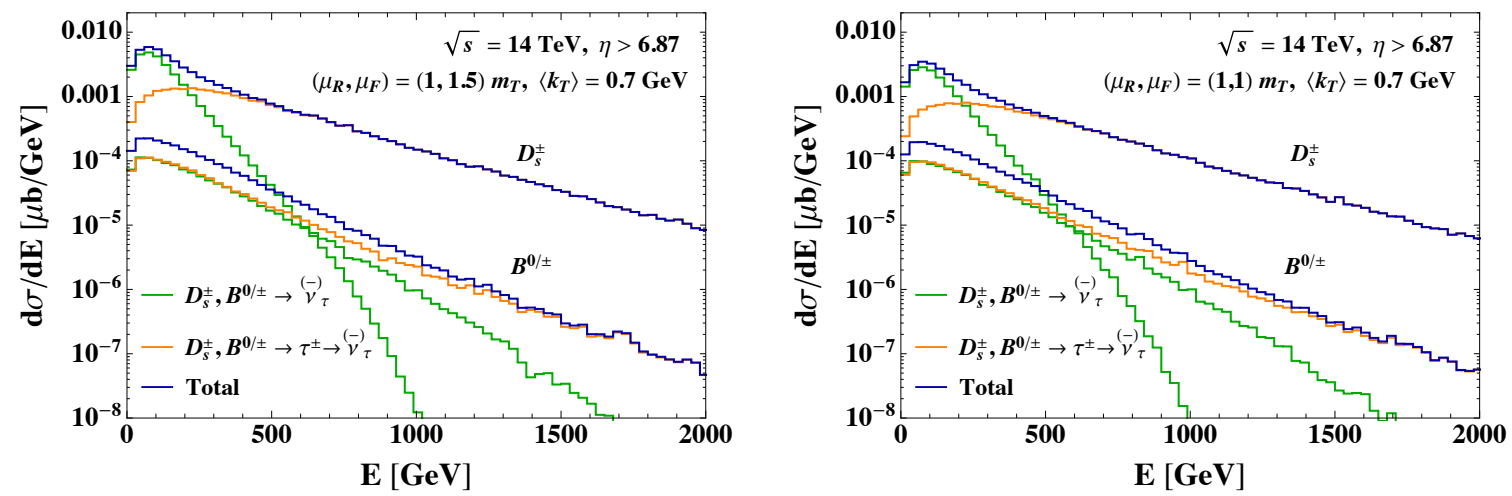

Figure 4. The neutrino energy distributions for tau neutrinos and antineutrinos from the direct decay $D_{s}^{ \pm} / B^{0, \pm} \rightarrow \stackrel{(-)}{\nu}$ (green) and the chain decay $D_{s}^{ \pm} / B^{0, \pm} \rightarrow \tau^{ \pm} \rightarrow \stackrel{(-)}{\nu} \tau$ (orange) and their sum (blue) for neutrinos with pseudorapidity $\eta>6.87$, produced in $p p$ collisions at $\sqrt{s}=14 \mathrm{TeV}$. Predictions are obtained using as input $\left\langle k_{T}\right\rangle=0.7 \mathrm{GeV}$ with our default scale combination $\left(\mu_{R}, \mu_{F}\right)=(1.0,1.5) m_{T}$ (left) and with the conventional scale combination $\left(\mu_{R}, \mu_{F}\right)=(1.0,1.0) m_{T}$ (right). The contributions from both $D_{s}$ and $B$ mesons are shown separately.

all the branching fractions. The energy and angular distributions of tau neutrinos from $B$ meson decays are discussed in appendix A, as are the energy and angular distributions of muon and electron neutrinos from heavy-meson decays.

Figure 4 shows the energy distributions for tau neutrinos and antineutrinos from both the $D_{s}$ and $B$ meson decays with neutrino pseudorapidity $\eta>6.87$. The contribution of the direct and the chain decays are shown separately, as well as their sum. The left panel shows the distributions with our default scale combination $\left(\mu_{R}, \mu_{F}\right)=(1.0,1.5) m_{T}$, while the right panel shows the same distributions, but using as input $\left(\mu_{R}, \mu_{F}\right)=(1.0,1.0) m_{T}$. Qualitatively, the distributions are similar, although, as expected from the discussion in section 3, the differential distributions using the conventional scale are lower than with our default scale choice.

The number of neutrino events per unit energy can be written as

$$
\frac{d N}{d E}=\frac{d \sigma(p p \rightarrow \nu X)}{d E} \times \mathcal{P}_{\text {int }} \times \mathcal{L},
$$

where the interaction probability in the detector is

$$
\mathcal{P}_{\text {int }}=\left(\rho_{\mathrm{Pb}} \times l_{\text {det }} \times N_{\mathrm{avo}}\right) \frac{\sigma_{\nu_{\tau} P b}}{A_{\mathrm{Pb}}} .
$$

Here, we use an integrated luminosity $\mathcal{L}=3000 \mathrm{fb}^{-1}$ and a lead density $\rho_{P b}=11.34 \mathrm{~g} / \mathrm{cm}^{3}$. The nucleon number of lead is $A_{P b}=208$ and $l_{\text {det }}$ is the length of the lead neutrino target in the detector which is also characterized by a cross sectional area of radius $1 \mathrm{~m}$ (thus $\eta>6.87$ ) for our discussion here. For reference, a detector of lead with radius of $1 \mathrm{~m}$ and length $l_{\text {det }}=1 \mathrm{~m}$ has a mass of $\sim 35.6$ ton. For the number of events, we quote the number of events per ton of lead (per $2.8 \mathrm{~cm}$ depth of a lead disk with radius $1 \mathrm{~m}$ ). As discussed in more detail in ref. [62], we evaluate the neutrino and antineutrino charged-current cross sections at NLO, including mass effects [63-67], using the nCTEQ15 PDFs for lead [47]. For 

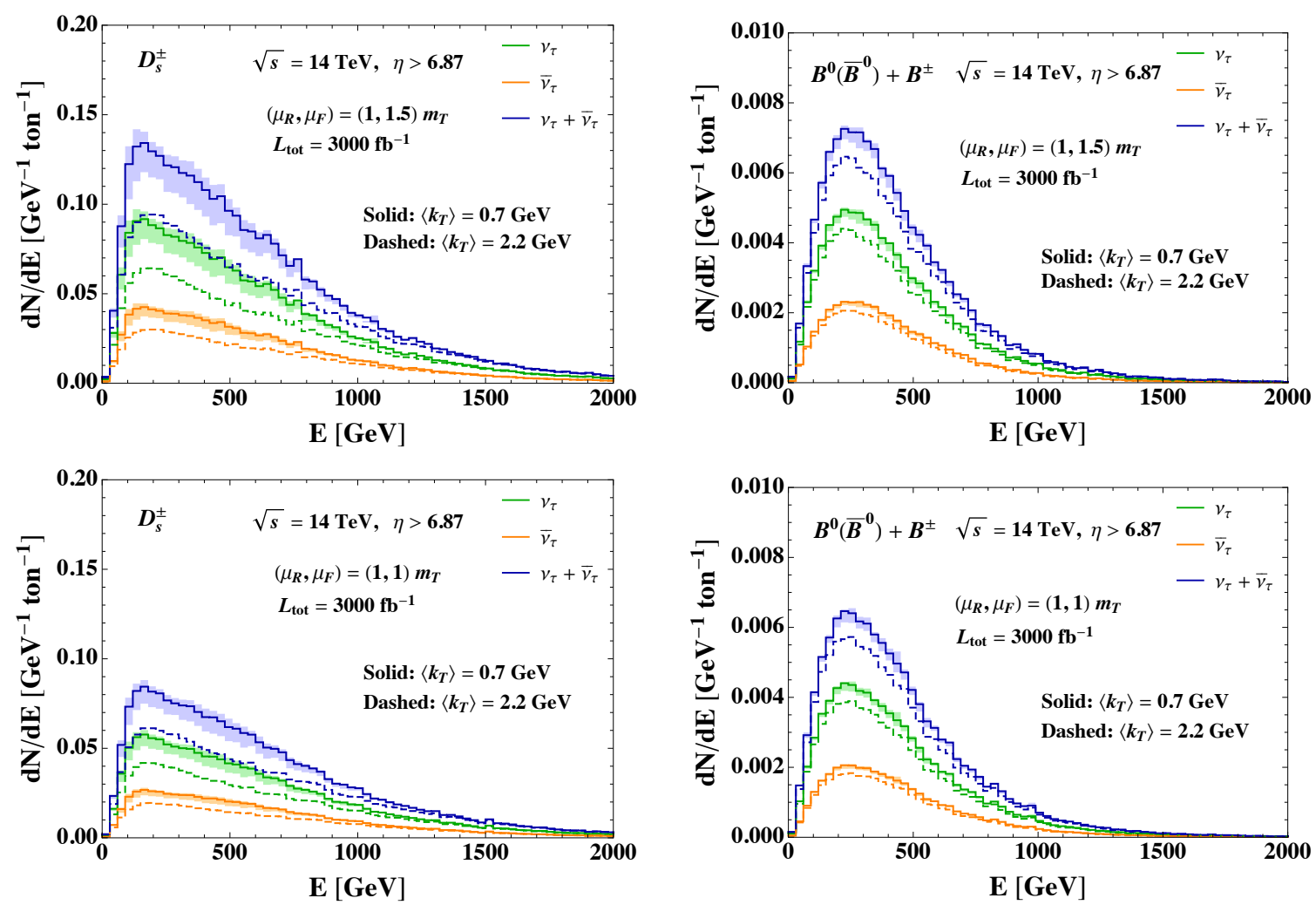

Figure 5. Our predictions for the tau neutrino and/or antineutrino number of charged-current events per $\mathrm{GeV}$ per ton as a function of the incident neutrino energy for neutrino pseudorapidity $\eta>6.87$ for $p p$ collisions with $\sqrt{s}=14 \mathrm{TeV}$ and integrated luminosity $\mathcal{L}=3000 \mathrm{fb}^{-1}$. The central predictions (solid histogram) refer to the $\left\langle k_{T}\right\rangle=0.7 \mathrm{GeV}$ value and the bands arise from the $\left\langle k_{T}\right\rangle$ variation in the range $0<\left\langle k_{T}\right\rangle<1.4 \mathrm{GeV}$. The results obtained with $\left\langle k_{T}\right\rangle=2.2 \mathrm{GeV}$ are also shown (dashed histogram). The upper plots are obtained by setting the QCD scales to $\left(\mu_{R}, \mu_{F}\right)=(1.0,1.5) m_{T}$, while the lower plots refer to the conventional scale combination $\left(\mu_{R}, \mu_{F}\right)=(1.0,1.0) m_{T}$. NLO corrections are included in the DIS cross sections.

neutrino energies above $\sim 10 \mathrm{GeV}$, deep inelastic scattering (DIS) dominates quasi-elastic scattering and few-pion production $[68,69]$. At the energies of interest, the neutrino DIS cross section is roughly a factor of $\sim 2$ larger than the antineutrino cross section. Kinematic corrections due to the tau mass reduce the charged-current cross section by $\sim 25 \%$ for a neutrino energy $E_{\nu}=100 \mathrm{GeV}$, and by $\sim 5 \%$ for $E_{\nu}=1000 \mathrm{GeV}$ [66]. NLO corrections are small, less than $\sim 5 \%$ for neutrino energies of $100 \mathrm{GeV}$, as shown e.g. in ref. [65].

In figure 5, we show the number of events per unit neutrino energy per ton of detector lead for tau neutrinos and antineutrinos from the $D_{s}^{ \pm}$(left panels) and $B$ meson (right panels) decays, evaluated for a total integrated luminosity $\mathcal{L}=3000 \mathrm{fb}^{-1}$. The upper panels include results with our default scales $\left(\mu_{R}, \mu_{F}\right)=(1.0,1.5) m_{T}$, while the lower panels show the same quantities using as input the conventional scale combination $\left(\mu_{R}, \mu_{F}\right)=$ $(1.0,1.0) m_{T}$. The central solid histograms are obtained for $\left\langle k_{T}\right\rangle=0.7 \mathrm{GeV}$, and the bands reflect the uncertainty range due to the variation of $\left\langle k_{T}\right\rangle$ in the range $0-1.4 \mathrm{GeV}$. We also present predictions for $\left\langle k_{T}\right\rangle=2.2 \mathrm{GeV}$, that are shown with the dashed histograms. 
Incorporating $\left\langle k_{T}\right\rangle$ effects has a large impact on the predictions at low energies as expected from figure 2. In particular, computing charm production with $\left\langle k_{T}\right\rangle=0 \mathrm{GeV}$, enhances the number of events per unit energy by up to $\sim 8 \%$ at $E_{\nu} \sim 100 \mathrm{GeV}$ with respect to the case with the default $\left\langle k_{T}\right\rangle=0.7 \mathrm{GeV}$, whereas the differences are less than $1 \%$ for $E_{\nu} \gtrsim 1000 \mathrm{GeV}$. The lower limit on the number of events per unit energy, corresponding to the case $\left\langle k_{T}\right\rangle=1.4 \mathrm{GeV}$, is lower by $\sim 16(5) \%$ at $E_{\nu} \sim 100(1000) \mathrm{GeV}$ with respect to the case with $\left\langle k_{T}\right\rangle=0.7 \mathrm{GeV}$, whereas the difference reduces to $1 \%$ or even less for $E_{\nu} \gtrsim$ $1500 \mathrm{GeV}$. On the other hand, if $\left\langle k_{T}\right\rangle=2.2 \mathrm{GeV}$, the number of events from $D_{s}^{ \pm}$in the peak of the distribution is a factor of $\sim 2 / 3$ lower than in the peak for $\left\langle k_{T}\right\rangle=0.7 \mathrm{GeV}$. The $\left\langle k_{T}\right\rangle$ sensitivity of the predictions of the number of $\left(\nu_{\tau}+\bar{\nu}_{\tau}\right)$ neutrinos from $B$ meson decays is much smaller than for $D_{s}$ meson decays. A comparison of upper and lower panels shows the stronger impact of the factorization scale dependence on the predictions for $\nu_{\tau}+\bar{\nu}_{\tau}$ from charm mesons than from $B$ mesons.

Figure 6 shows the uncertainty bands associated with the QCD scale variation in the range considered in figure 1 , for a central scale choice $\left(\mu_{R}, \mu_{F}\right)=(1,1.5) m_{T}$. These bands are computed as envelopes of seven combinations of $\left(N_{R}, N_{F}\right)$, equal to factors of $m_{T}$ of $(0.5,0.75),(2.0,3.0),(1.0,0.75),(0.5,1.5),(1.0,3.0),(2.0,1.5)$ and $(1.0,1.5)$. For neutrinos from the $D_{s}$ decay, the upper boundary of the band is larger than the central prediction by a factor of $\sim 3-4$, while the lower edge of the band is $40-60 \%$ smaller than the central prediction for $E_{\nu} \lesssim 1500 \mathrm{GeV}$. Thus, the QCD scale uncertainty band in our evaluation with $\left\langle k_{T}\right\rangle=0.7 \mathrm{GeV}$ has overlap with the prediction using $\left\langle k_{T}\right\rangle=2.2 \mathrm{GeV}$, as follows from comparing figures 5 and 6 . For neutrinos from $B$ meson decays, the scale uncertainty bands are smaller than for neutrinos from $D$ mesons. In particular, the edge of the upper uncertainty band is a factor 1.5-2 larger than the central prediction, whereas the lower uncertainty band extends to about $20 \%$ below the central prediction for $E_{\nu} \lesssim$ $1000 \mathrm{GeV}$. The scale uncertainty bands around central predictions with the conventional scale combination $\left(\mu_{R}, \mu_{F}\right)=(1.0,1.0) m_{T}$, as shown in figure 7 , have similar sizes to those in figure 6 . However, the conventional scale choice leads to an overall lower central prediction for the number of events from both $D_{s}$ and $B \rightarrow \nu_{\tau}$ and $\bar{\nu}_{\tau}$ than the case $\left(\mu_{R}, \mu_{F}\right)=(1.0,1.5) m_{T}$, as follows by comparing figures 6 and 7 .

In tables 1 and 2, we present the total event numbers for our preferred scale choice $\left(\mu_{R}, \mu_{F}\right)=(1.0,1.5) m_{T}$ and the conventional QCD scale choice $\left(\mu_{R}, \mu_{F}\right)=(1.0,1.0) m_{T}$, respectively, for $1 \mathrm{~m}$ of lead (35.6 ton). Assuming an integrated luminosity of $3,000 \mathrm{fb}^{-1}$, each table shows separately the number of $\nu_{\tau}$ and $\bar{\nu}_{\tau}$ events from $D_{s}^{ \pm}$and $B$ meson decays, respectively, and the total number, each for selected $\left(\mu_{R}, \mu_{F}\right)$ scale and $\left\langle k_{T}\right\rangle$ choices. Table 1 shows that when using $\left(\mu_{R}, \mu_{F}\right)=(1.0,1.5) m_{T}$ and the $\left\langle k_{T}\right\rangle$ value that yields the best match to the POWHEG + PYTHIA $D_{s}^{ \pm}$energy distribution in the far forward $\eta>6.87$ region $\left(\left\langle k_{T}\right\rangle=0.7 \mathrm{GeV}\right)$, the total number of tau neutrino plus antineutrino events is predicted to be $\sim 3,800$. Factorization and renormalization scale variations around the QCD central scales yield a very broad uncertainty band on the number of events, varying in the interval $\sim 1,800-11,200$. The large scale uncertainties indicate that contributions from missing higher orders in the perturbative calculation in collinear factorization are relatively large. On the other hand, the central scale choice, with $\left\langle k_{T}\right\rangle$ variation in the range 

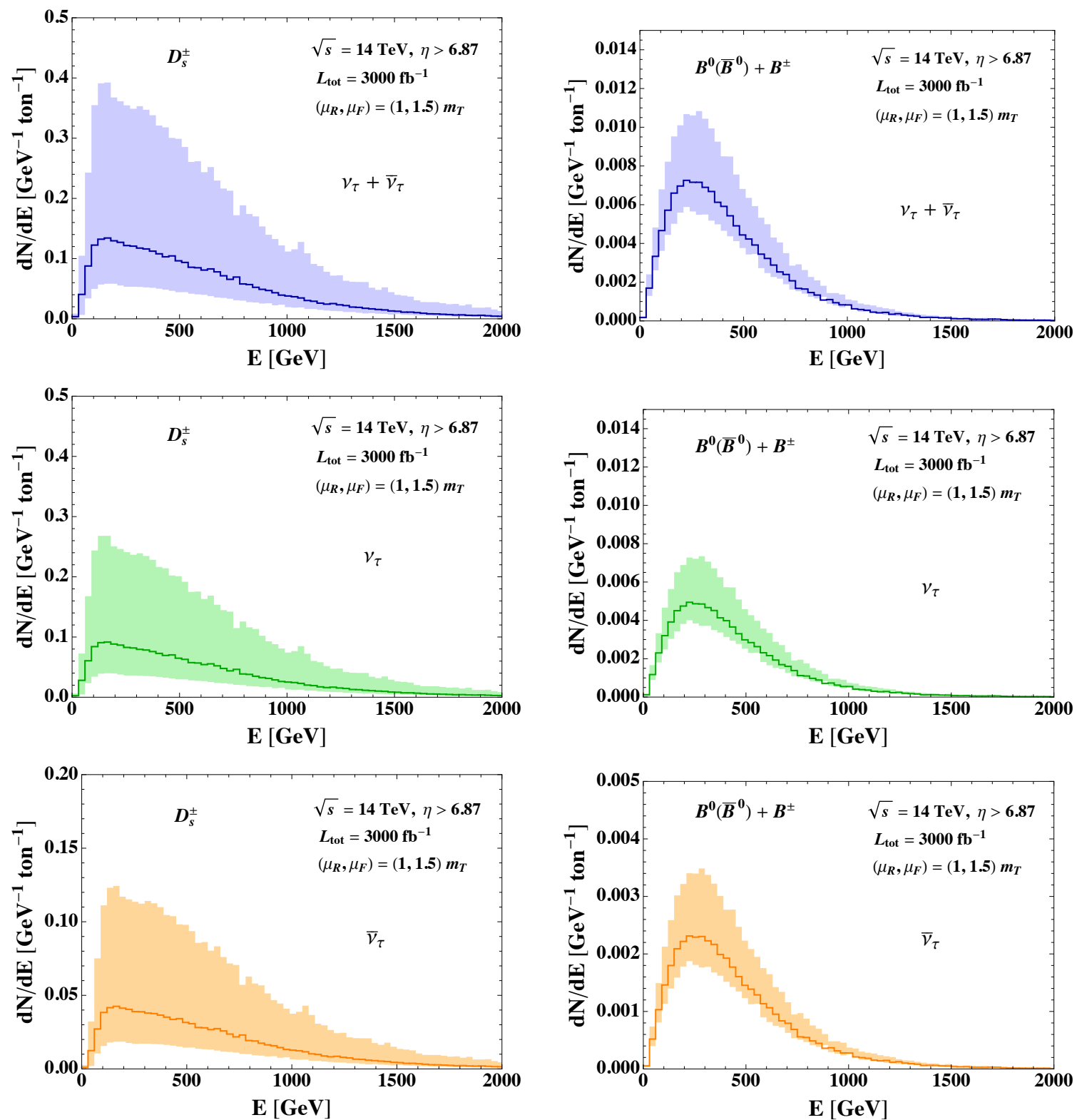

Figure 6. Uncertainty range due to the QCD scale variation in the tau neutrino and antineutrino number of charged-current events per $\mathrm{GeV}$ per ton as a function of the incident neutrino energy for neutrino pseudorapidity $\eta>6.87$ in $p p$ collisions with $\sqrt{s}=14 \mathrm{TeV}$ and integrated luminosity $\mathcal{L}=3000 \mathrm{fb}^{-1}$. The central predictions are obtained using as input $\left(\mu_{R}, \mu_{F}\right)=(1,1.5) m_{T}$ and $\left\langle k_{T}\right\rangle=0.7 \mathrm{GeV}$. The upper and lower limits arise from the QCD 7-point scale variation range in figure 1. NLO QCD corrections are accounted for in the DIS pQCD cross section.

$\left\langle k_{T}\right\rangle=0-2.2 \mathrm{GeV}$, produces a smaller uncertainty in the number of events, which span the range $\sim 3,900-2,900$, with the lower end of the range of number of events corresponding to the $\left(\mu_{R}, \mu_{F}\right.$ and $\left.\left\langle k_{T}\right\rangle\right)$-combination that is favored by LHCb data. Given our expectation that, for $\left\langle k_{T}\right\rangle=2.2 \mathrm{GeV}$, some effects of missing higher-order perturbative corrections are compensated by the large value of this parameter, an uncertainty in $\left\langle k_{T}\right\rangle$ cannot be added 

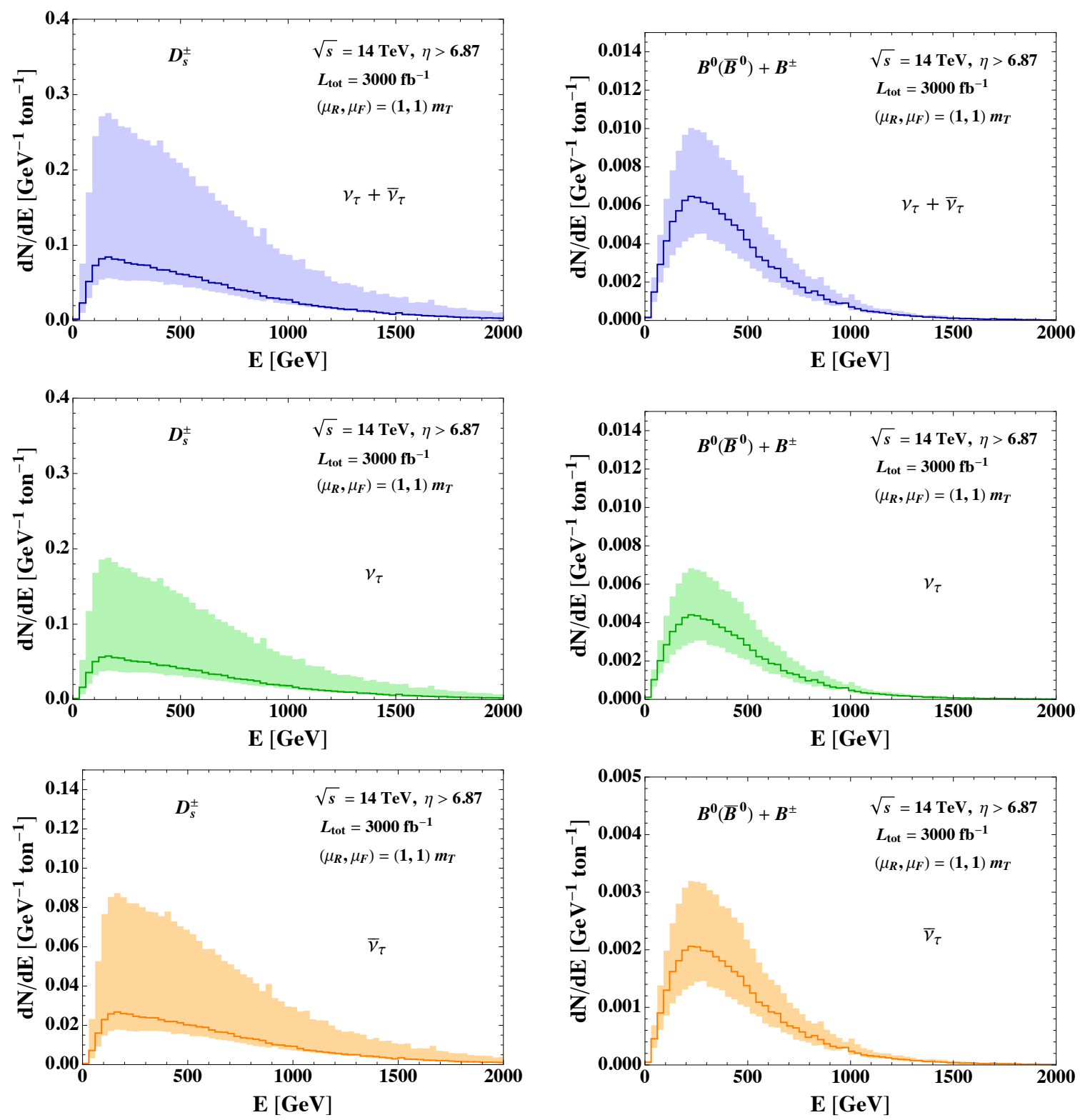

Figure 7. The same as figure 6, but adopting the central scale conventional choice $\left(\mu_{R}, \mu_{F}\right)=$ $(1.0,1.0) m_{T}$ and seven-point scale variation around it, again with $\left\langle k_{T}\right\rangle=0.7 \mathrm{GeV}$.

to the scale uncertainty to get a total uncertainty for the number of events. In any case, the scale uncertainty yields a range of number of events that is significantly larger than the range found by varying $\left\langle k_{T}\right\rangle$ from 0 to $2.2 \mathrm{GeV}$.

We set $\left(\mu_{R}, \mu_{F}\right)=(1.0,1.0) m_{T}$, the conventional scales used in most QCD theory evaluations of heavy-flavor production, for the predictions in table 2. This table shows as well a broad range of predicted number of events, with a central value of $\sim 2,500$ events for $\left\langle k_{T}\right\rangle=0.7 \mathrm{GeV}$. This number amounts to a factor of $\sim 2 / 3$ of the one obtained in the evaluation with $\left(\mu_{R}, \mu_{F}\right)=(1,1.5) m_{T}$ for $\left\langle k_{T}\right\rangle=0.7 \mathrm{GeV}$, however, it is comparable to the 2,900 events for $\left\langle k_{T}\right\rangle=2.2 \mathrm{GeV}$ with $\left(\mu_{R}, \mu_{F}\right)=(1,1.5) m_{T}$. 


\begin{tabular}{|c|c|c|c|c|c|c|c|c|}
\hline & $\nu_{\tau}$ & $\bar{\nu}_{\tau}$ & $\nu_{\tau}+\bar{\nu}_{\tau}$ & \multicolumn{5}{c|}{$\nu_{\tau}+\bar{\nu}_{\tau}$} \\
\hline$\left(\mu_{R}, \mu_{F}\right)$ & \multicolumn{3}{|c|}{$(1,1.5) m_{T}$} & \multicolumn{3}{c|}{$(1,1.5) m_{T}$} & $(0.5,1.5) m_{T}$ & $(1,0.75) m_{T}$ \\
\hline$\left\langle k_{T}\right\rangle$ & \multicolumn{3}{|c|}{$0.7 \mathrm{GeV}$} & $0 \mathrm{GeV}$ & $1.4 \mathrm{GeV}$ & $2.2 \mathrm{GeV}$ & \multicolumn{2}{c|}{$0.7 \mathrm{GeV}$} \\
\hline$D_{s}$ & 2451 & 1191 & 3642 & 3799 & 3261 & 2735 & 11008 & 1716 \\
\hline$B^{ \pm, 0}$ & 96 & 46 & 142 & 144 & 137 & 127 & 214 & 115 \\
\hline Total & 2547 & 1237 & 3784 & 3943 & 3398 & 2862 & 11222 & 1831 \\
\hline
\end{tabular}

Table 1. The charged-current event numbers for tau neutrinos and antineutrinos in $1 \mathrm{~m}$ length of the lead detector (equivalent to $M_{\mathrm{pb}} \simeq 35.6$ ton) assuming central scales $\left(\mu_{R}, \mu_{F}\right)=(1.0,1.5) m_{T}$ in the computation of heavy-meson production in $p p$ collisions at $\sqrt{s}=14 \mathrm{TeV}$ and an integrated luminosity $\mathcal{L}=3000 \mathrm{fb}^{-1}$. The scale combinations $(0.5,1.5) m_{T}$ and $(1,0.75) m_{T}$ give the upper and lower limits of the scale variation envelope obtained from the seven combinations of $\left(N_{R}, N_{F}\right)$ shown in figure 6.

\begin{tabular}{|c|c|c|c|c|c|c|c|c|}
\hline & $\nu_{\tau}$ & $\bar{\nu}_{\tau}$ & $\nu_{\tau}+\bar{\nu}_{\tau}$ & \multicolumn{5}{c|}{$\nu_{\tau}+\bar{\nu}_{\tau}$} \\
\hline$\left(\mu_{R}, \mu_{F}\right)$ & \multicolumn{3}{|c|}{$(1,1) m_{T}$} & \multicolumn{3}{c|}{$(1,1) m_{T}$} & $(0.5,1) m_{T}$ & $(1,0.5) m_{T}$ \\
\hline$\left\langle k_{T}\right\rangle$ & \multicolumn{3}{|c|}{$0.7 \mathrm{GeV}$} & $0 \mathrm{GeV}$ & $1.4 \mathrm{GeV}$ & $2.2 \mathrm{GeV}$ & \multicolumn{2}{c|}{$0.7 \mathrm{GeV}$} \\
\hline$D_{s}$ & 1591 & 774 & 2365 & 2455 & 2143 & 1822 & 7834 & 1779 \\
\hline$B^{ \pm, 0}$ & 87 & 42 & 129 & 131 & 124 & 115 & 202 & 91 \\
\hline Total & 1678 & 816 & 2494 & 2586 & 2267 & 1937 & 8036 & 1870 \\
\hline
\end{tabular}

Table 2. Same as table 1, but adopting $\left(\mu_{R}, \mu_{F}\right)=(1.0,1.0) m_{T}$ as central scales in the computation of heavy-meson hadroproduction (conventional scale choice). The scale combinations $(0.5,1) m_{T}$ and $(1,0.5) m_{T}$ give the upper and lower limits of the scale variation envelope from the seven combinations of $\left(N_{R}, N_{F}\right)$ shown in figure 7 .

\subsection{Muon neutrinos}

At the interaction region, the muon neutrino and electron neutrino fluxes from heavyflavor production and decay will be nearly the same, coming primarily from the neutral and charged $D$ semileptonic decays. Figure 8 shows the energy distributions of the sum of muon neutrinos and antineutrinos. The upper two panels are for $\left(\mu_{R}, \mu_{F}\right)=(1.0,1.5) m_{T}$ and the lower two panels are for $\left(\mu_{R}, \mu_{F}\right)=(1.0,1.0) m_{T}$. The left plots show the contributions from the charm hadrons, $D^{+}, D^{0}, D_{s}^{+}$and $\Lambda_{c}$ and their sum. In the right plots, the total contributions from all charm hadrons and the bottom hadrons are shown. As can be seen in the left plots, the decays of $D^{ \pm}, D^{0}$ and $\bar{D}^{0}$ dominate the muon neutrino and antineutrino distributions. This is due to larger fragmentation fractions and decay branching fractions to muon neutrinos compared to those of $D_{s}^{ \pm}$and $\Lambda_{c}$. Similarly, the muon neutrinos and antineutrinos from $B$ meson decays are mainly from $B^{ \pm}, B^{0}$ and $\bar{B}^{0}$. The bottom hadron contributions, compared to the charm hadron contributions, to the inclusive muon neutrino plus antineutrino energy distribution at $\sqrt{s}=14 \mathrm{TeV}$ with $\eta>6.87$ is about a factor $\sim 1 / 60$ smaller than the distributions from charm at low energy, and a 

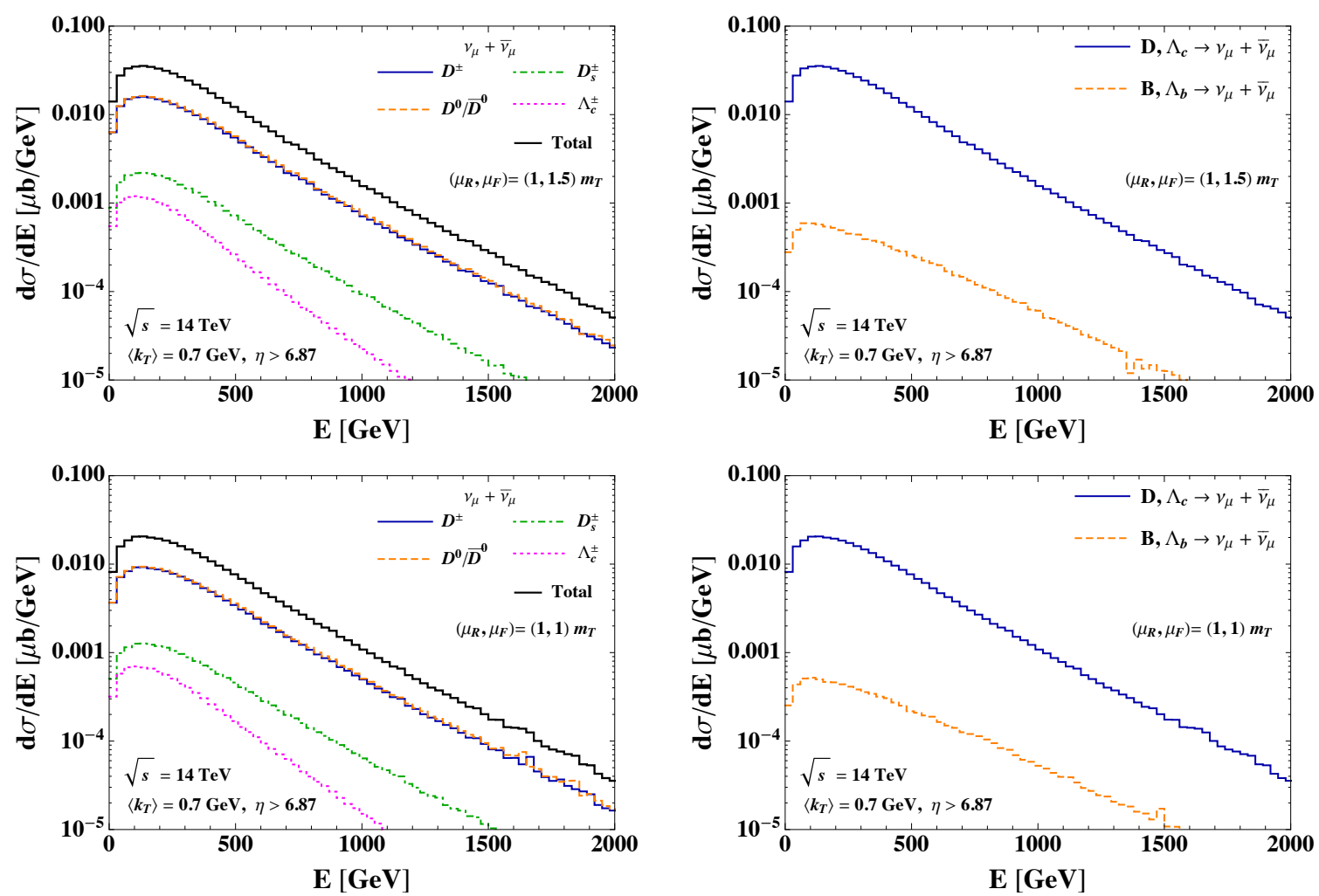

Figure 8. Our predictions for the muon neutrinos plus antineutrinos energy distribution for $p p$ collisions at $\sqrt{s}=14 \mathrm{TeV}$, with neutrino pseudorapidity $\eta>6.87$. Left: shown are the contributions from each charm hadron $D^{+}, D^{0}, D_{s}^{+}$and $\Lambda_{c}$ and their antiparticles, together with their sum. Right: total contributions from the charm hadrons and bottom hadrons are presented, respectively. The total for $B$-hadrons accounts for the contributions from $B^{+}, B^{0}, B_{s}^{+}$and $\Lambda_{b}$ and their antiparticles. The upper plots refer to the scale choice $\left(\mu_{R}, \mu_{F}\right)=(1.0,1.5) m_{T}$, while the lower plots correspond to the conventional choice $\left(\mu_{R}, \mu_{F}\right)=(1.0,1.0) m_{T}$. The value $\left\langle k_{T}\right\rangle=0.7 \mathrm{GeV}$ is used as input in all cases.

factor of $\sim 1 / 20$ at high energy. As in figure 4 for $\nu_{\tau}+\bar{\nu}_{\tau}$, figure 8 shows that the predicted energy distribution of $\nu_{\mu}+\bar{\nu}_{\mu}$ from charm is $\sim 1.5$ larger for $\left(\mu_{R}, \mu_{F}\right)=(1.0,1.5) m_{T}$ than for $\left(\mu_{R}, \mu_{F}\right)=(1.0,1.0) m_{T}$, while the smaller contributions from $B$ hadrons are much less sensitive to the scale choice.

The corresponding predictions for the number of muon neutrino and antineutrino charged-current events per unit energy for a 1 ton lead target for $\eta>6.87$ are shown in figures 9 and 10. Figure 9 shows the number of muon neutrino and muon antineutrino events per unit energy from heavy flavor, including uncertainty bands from $\left\langle k_{T}\right\rangle$ variation in the range $[0,1.4] \mathrm{GeV}$ for $\left(\mu_{R}, \mu_{F}\right)=(1.0,1.5) m_{T}$. Again, the dashed histograms correspond to results with $\left\langle k_{T}\right\rangle=2.2 \mathrm{GeV}$. The left panels in figure 10 show, from top to bottom, the sum of $\nu_{\mu}+\bar{\nu}_{\mu}$ charged current events per ton of lead, for $\nu_{\mu}$ and for $\bar{\nu}_{\mu}$, all for $\left(\mu_{R}, \mu_{F}\right)=(1.0,1.5) m_{T}$. The right panels show the same, but with $\left(\mu_{R}, \mu_{F}\right)=(1.0,1.0) m_{T}$. Each panel includes a wide uncertainty band reflecting the theoretical uncertainties associated with scale variation. Our evaluation using NLO perturba- 

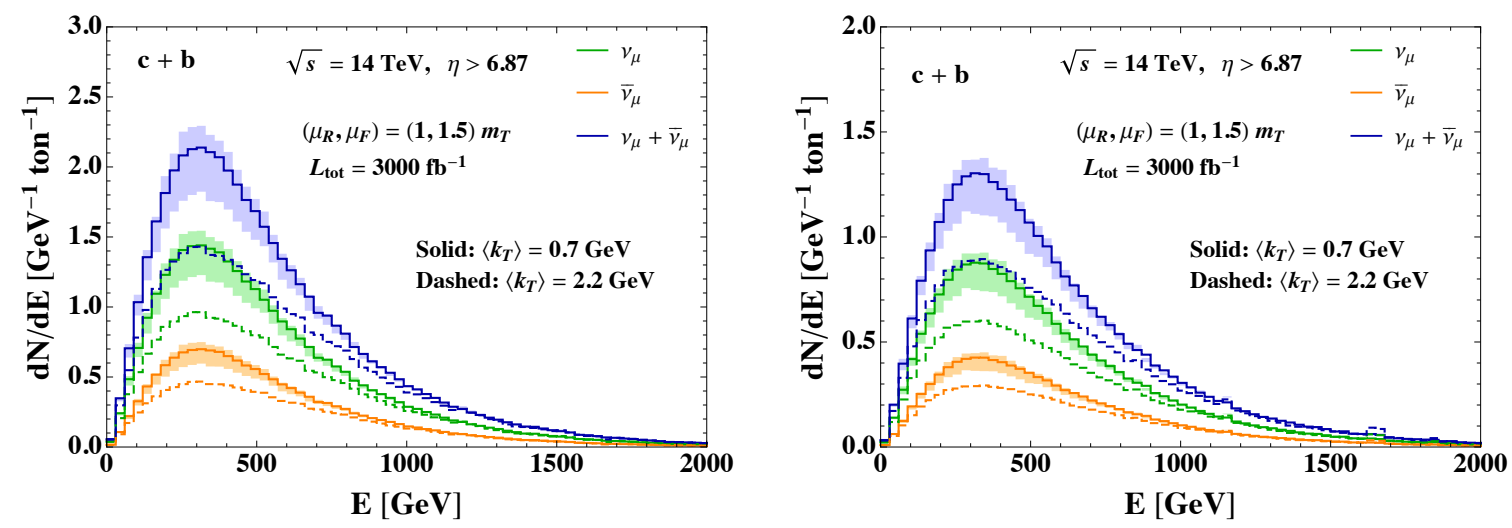

Figure 9. Our predictions for the muon neutrino and antineutrino number of charged current events per $\mathrm{GeV}$ for 1 ton of lead target as a function of the incident neutrino energy for neutrinos with a pseudorapidity $\eta>6.87$ generated by heavy-flavor decays in $p p$ collisions with $\sqrt{s}=14 \mathrm{TeV}$. A value of $\left\langle k_{T}\right\rangle=0.7 \mathrm{GeV}$ is adopted for the central predictions, while the uncertainty bands accounts for the effect of $\left\langle k_{T}\right\rangle$ variation in the interval $0-1.4 \mathrm{GeV}$. NLO QCD corrections are accounted for in the DIS cross section. The integrated luminosity amounts to $\mathcal{L}=3000 \mathrm{fb}^{-1}$.

tive QCD gives a factor of $\sim 13$ in the ratio of $\nu_{\mu}+\bar{\nu}_{\mu}$ to $\nu_{\tau}+\bar{\nu}_{\tau}$ events based on heavy flavor alone, with our default input parameters.

Heavy-flavor hadron decays, however, are not the only sources of $\nu_{\mu}+\bar{\nu}_{\mu}$. In principle, pion, kaon and weak gauge boson decays can also contribute. In ref. [10], the contributions from $W$ and $Z$ production and decay to neutrinos are studied. They show that neutrinos from weak gauge boson decays populate mostly the pseudorapidity range of $|\eta|<4$.5, but are negligible in the region $\eta>6.5$ [10].

At first glance, one might expect that pion and kaon decays will not be important sources of neutrinos. Pions with $E_{\pi}>9 \mathrm{GeV}$ have $\gamma c \tau>500 \mathrm{~m}$. Charged kaons have $\gamma c \tau>500 \mathrm{~m}$ for $E_{K}>67 \mathrm{GeV}$. Pion and kaon contributions to the number of $\nu_{\mu}+\bar{\nu}_{\mu}$ at high energy were neglected in the earlier work of ref. [14]. Park, in ref. [5], used PyтнiA to account for pions and kaons, requiring the decay to occur within $50 \mathrm{~m}$ of the interaction point to guarantee that particles decay inside the beam pipe, leading to a factor of $\sim 100$ times more $\nu_{\mu}+\bar{\nu}_{\mu}$ events than $\nu_{\tau}+\bar{\nu}_{\tau}$ events.

In ref. [18], a more detailed evaluation of $\nu_{\mu}+\bar{\nu}_{\mu}$ events is performed for a detector with $25 \times 25 \mathrm{~cm}^{2}$ cross-sectional area at 480 meters from the ATLAS interaction point. They find a factor of $\sim 1000$ more interactions by $\nu_{\mu}+\bar{\nu}_{\mu}$ than by $\nu_{\tau}+\bar{\nu}_{\tau}$ when pions and kaons are included, although with a different energy distribution. Their evaluation of heavy-flavor contributions is done using PYтнIA [17], while their light hadron production is estimated using the CRMC [70] simulation package with Epos-LHC [71], QGSJET-II-04 [72] and SibYLl 2.3c [73-75]. They find that most of the neutrinos with energies above $1 \mathrm{TeV}$ from charged light hadron decays come from pion and kaon decays in a region within $\sim 55 \mathrm{~m}$ from the ATLAS interaction point. They find that magnetic fields sweep lower-energy (below $\sim 100 \mathrm{GeV}$ ) charged particles away if the particles have travelled $20 \mathrm{~m}$ downstream from the interaction point and have passed through the front quadrupole absorber of inner 

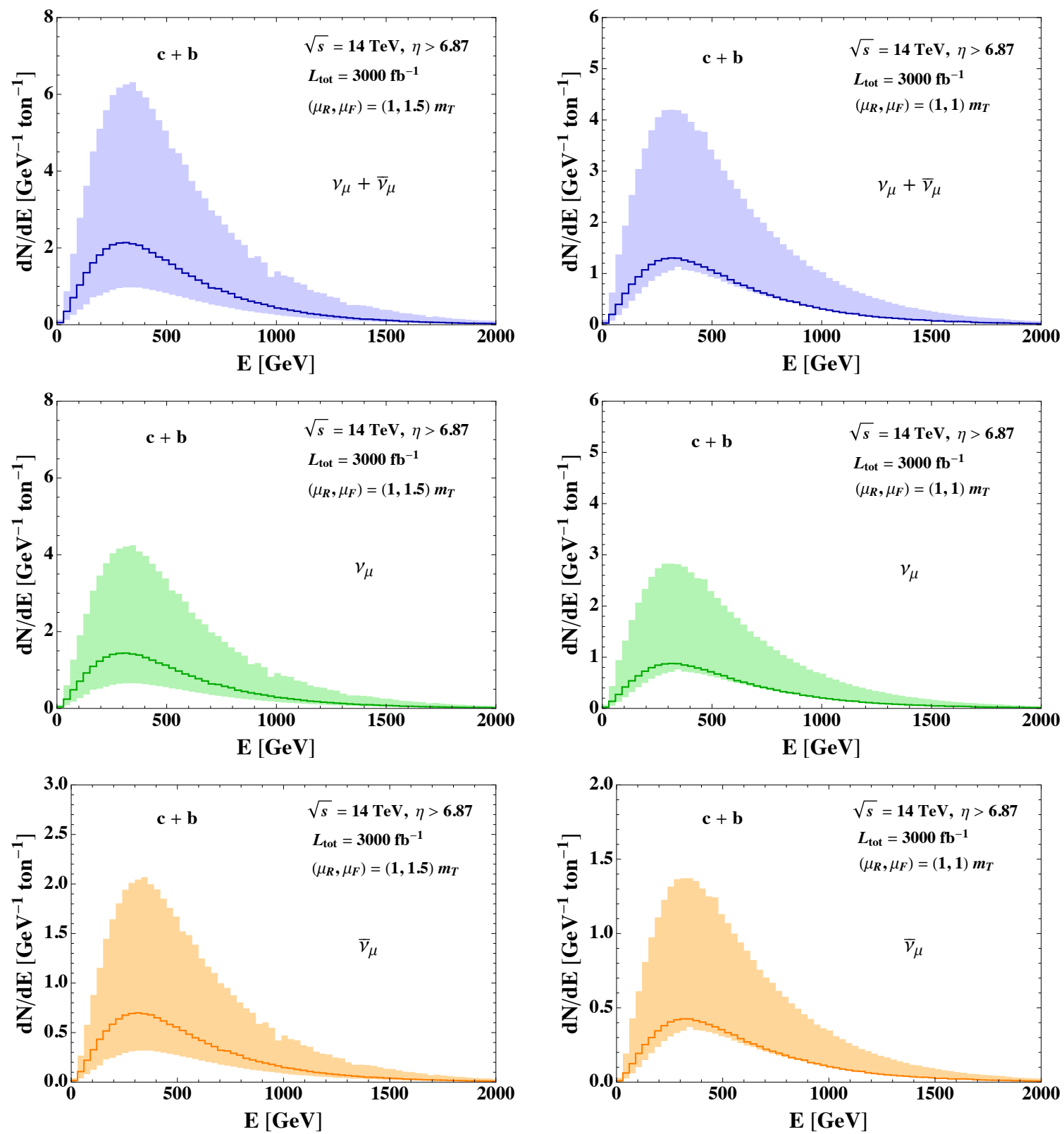

Figure 10. Uncertainty range due to the QCD scale variation in the muon neutrino and antineutrino number of charged current events per $\mathrm{GeV}$ per ton as a function of the incident neutrino energy for neutrinos with pseudorapidity $\eta>6.87$ generated by heavy-flavor decays in $p p$ collisions with $\sqrt{s}=14 \mathrm{TeV}$ and integrated luminosity $\mathcal{L}=3000 \mathrm{fb}^{-1}$. The central predictions are obtained using as input $\left(\mu_{R}, \mu_{F}\right)=(1,1.5) m_{T}$ (left) and $\left(\mu_{R}, \mu_{F}\right)=(1.0,1.0) m_{T}$ (right), respectively. The upper and lower limits in each panel arise from 7 -point scale variation in the same range as in figure 1. 
radius $17 \mathrm{~mm}$ [18]. They also find that two-body decays of charged pions and charged kaons are the dominant sources of $\nu_{\mu}+\bar{\nu}_{\mu}$ production.

Our primary focus here is on the heavy-flavor contributions to the number of events from $\nu_{\mu}+\bar{\nu}_{\mu}$ and $\nu_{\tau}+\bar{\nu}_{\tau}$. Instead of simulating light charged hadron trajectories in magnetic fields, guided by the results of ref. [18], we approximately evaluate the number of $\nu_{\mu}+\bar{\nu}_{\mu}$ that come from pions and kaons as follows. We evaluate the $\pi^{ \pm}$and $K^{ \pm}$two-body decay contributions to the flux of $\nu_{\mu}+\bar{\nu}_{\mu}$ with the requirement that the mesons decay within $55 \mathrm{~m}$ of the interaction point and the decaying hadron's momentum lies within an angle of $\theta<1 \mathrm{mrad}$ relative to the beam axis to stay within the opening of the quadrupole absorber. While the 2-body light meson decays dominate, a more complete calculation of the light meson contributions to $\nu_{\mu}+\bar{\nu}_{\mu}$ would include $K_{L}$ semileptonic decays.

We use the parametrization of Koers et al. [34], based on fits to PYTHIA distributions for charged pions and kaons as a function of energy and rapidity, to generate the pion and kaon distributions. For reference, pion and kaon charged-particle multiplicities per interaction in $p p$ collisions at $\sqrt{s}=14 \mathrm{TeV}$ are $\sim 50$ and $\sim 6$, respectively [34]. Two-body decays are implemented, as for the $D_{s}^{ \pm} \rightarrow \nu_{\tau} \tau$ case, with the requisite changes to the initial and final particle masses. Compared to figure 8, charged pions and kaons contribute a factor of $\sim 100$ more $\nu_{\mu}+\bar{\nu}_{\mu}$ than heavy flavors do, as shown by the blue and red curves in figure 11. We will turn to the issue of the oscillations of neutrinos of different flavors to tau neutrinos. To estimate the number of $\nu_{e}+\bar{\nu}_{e}$, we consider the dominant contribution which is from $K_{L} \rightarrow \pi e \nu_{e}$. With the same geometry requirements, we show with the green curve in figure 11 the contribution from $K_{L}$ production and decay into $\nu_{e}+\bar{\nu}_{e}$. We use the Koers distributions for $K^{+}+K^{-}$, then divide by two for $K_{L}$. The three-body semileptonic kaon decay is evaluated following ref. [76]. The peak of the electron neutrino distribution from $K_{L}$ decays is about a factor of $\sim 2$ larger than the peak of the electron neutrino distribution from heavy-flavor decays. While $K_{L}$ semileptonic decays to $\nu_{e}$ are dominant, a more complete calculation would include the $K^{+} \rightarrow \nu_{e}$ semileptonic decays as well.

Three-flavor neutrino oscillations of the much larger number of $\nu_{\mu}+\bar{\nu}_{\mu}$ from charged pions and kaons to $\nu_{\tau}+\bar{\nu}_{\tau}$ could, in principle, overwhelm the number of $\nu_{\tau}+\bar{\nu}_{\tau}$ from heavyflavor decays. However, the baseline of $480 \mathrm{~m}$ is not at all optimal for $\nu_{\mu} \rightarrow \nu_{\tau}$ oscillations in the standard scenario with 3 active flavors. In two flavor approximation, for a mass squared difference of $\Delta m_{32}^{2} \simeq 2.5 \times 10^{-3} \mathrm{eV}^{2}$ and a mixing angle $\theta_{23} \simeq \pi / 4$, the oscillation probability is $P\left(\nu_{\mu} \rightarrow \nu_{\tau}\right) \simeq 2.3 \times 10^{-6} /\left(E^{2} / \mathrm{GeV}^{2}\right)$, so for energies above $E_{\nu_{\mu}}=10 \mathrm{GeV}$, $\nu_{\mu} \rightarrow \nu_{\tau}$ oscillations give a negligible contribution to the number of $\nu_{\tau}+\bar{\nu}_{\tau}$, even given the large theoretical uncertainties in the number of $\nu_{\tau}+\bar{\nu}_{\tau}$ events per unit energy. The $\nu_{e} \rightarrow \nu_{\tau}$ oscillation probability is even smaller.

In the next section, we use the approximate numbers of electron and muon neutrinos from light mesons, together with our heavy flavor results for all three neutrino flavors and turn to tau neutrino oscillations in a $3+1$ neutrino mixing framework. This illustrates an example of a signal of new physics that could be probed by a forward neutrino detector at the LHC when heavy-flavor uncertainties are under better theoretical control. While not necessary for an analysis of $\nu_{\tau}+\bar{\nu}_{\tau}$ because tau neutrinos come from $D_{s}$ and $B$ decays, for an analysis of all three neutrino flavors, a full accounting of light meson production and 


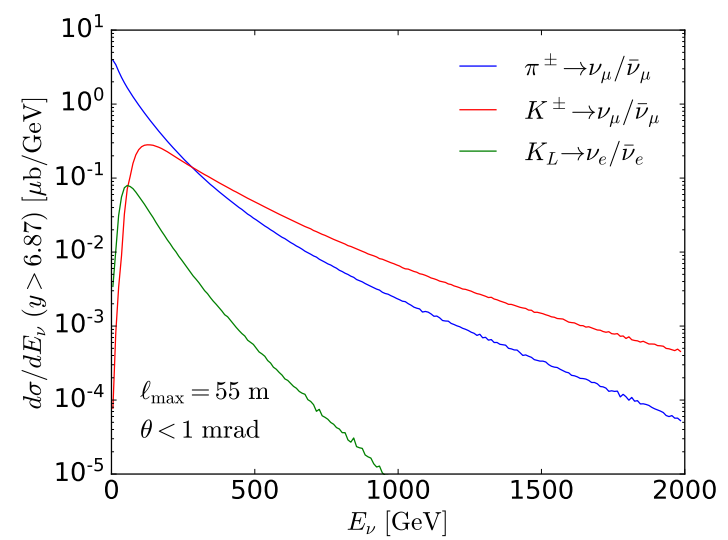

Figure 11. The $\nu_{\mu}+\bar{\nu}_{\mu}$ energy distributions from charged pions (blue) and kaons (red) and the $\nu_{e}+\bar{\nu}_{e}$ energy distributions from $K_{L}$ semileptonic decays (green) for forward neutrinos $(\eta>6.87)$ from the decay of mesons produced in $p p$ interactions at $\sqrt{s}=14 \mathrm{TeV}$, considering those mesons whose decay occurs within $55 \mathrm{~m}$ of the interaction point and whose momentum lies within an angle of $\theta<1 \mathrm{mrad}$ from the beam axis. The meson energy and rapidity distributions are evaluated using the parametrization of Koers et al. [34].

decay to neutrinos, using all available data on forward production (e.g., from LHCf [77], TOTEM [78] and CMS [79]) along with magnet and detector configurations in the interaction region, will be necessary. A forward tune of PyтніA that is underway [19] would also guide future work.

\section{New physics}

The detection of a large number of identifiable $\nu_{\tau}+\bar{\nu}_{\tau}$ events would offer opportunities to explore a new corner of parameter space for neutrino oscillations into a fourth "sterile" neutrino. The baseline of $480 \mathrm{~m}$ is most sensitive to a fourth neutrino mass $m_{4}$ of the order of tens of eV.

The flavor eigenstates $\nu_{l}$ can be expressed as a superposition of the mass eigenstates $\nu_{j}$ according to the formula

$$
\nu_{l}=\sum_{j=1}^{n_{\nu}} U_{l j} \nu_{j},
$$

where $n_{\nu}$ is the total number of neutrinos subject to oscillations: for the standard oscillation scenario with three active neutrinos, $n_{\nu}=3$, while for an oscillation scenario with three active neutrinos plus one sterile neutrino, $n_{\nu}=4$. The full transition probability appears in, for example, ref. [1]. The blue lines in the two panels of figure 12 show that tau neutrino survival probability $P\left(\nu_{\tau} \rightarrow \nu_{\tau}\right)$ in the three-flavor scenario approaches unity in the energy range $E_{\nu} \in[1,1000] \mathrm{GeV}$. The values of the parameters used here for three-flavor mixing are $\sin ^{2}\left(\theta_{12}\right)=0.310, \sin ^{2}\left(\theta_{13}\right)=0.02241, \sin ^{2}\left(\theta_{23}\right)=0.580, \Delta m_{21}^{2}=7.5 \times 10^{-5} \mathrm{eV}^{2}$ and $\Delta m_{31}^{2}=2.457 \times 10^{-3} \mathrm{eV}^{2}$ [80]. The values of all $\mathrm{CP}$ phases are taken to be zero. For a baseline of $480 \mathrm{~m}$, the tau neutrino survival probability has significant features in the few 

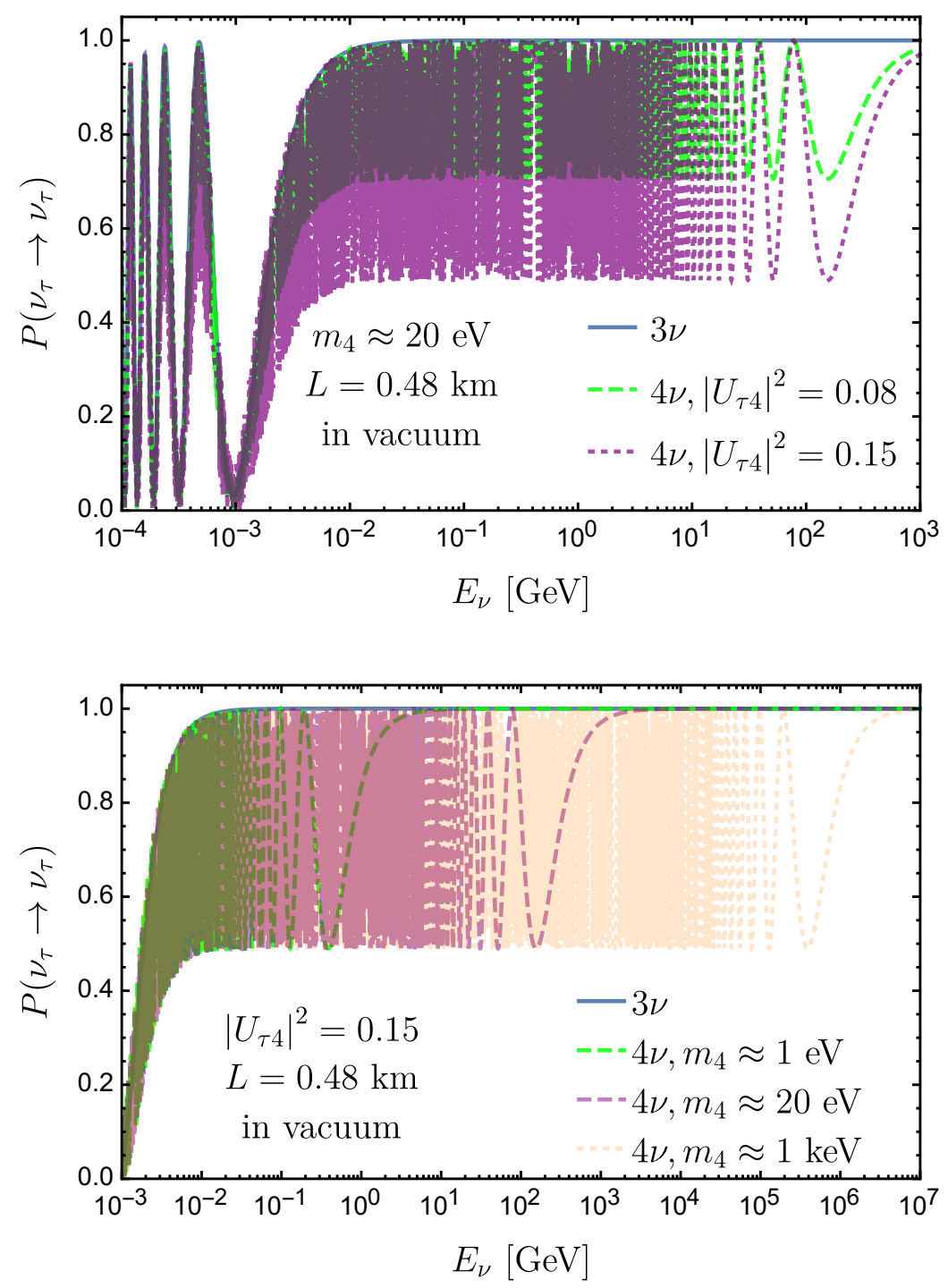

Figure 12. The survival probability $P\left(\nu_{\tau} \rightarrow \nu_{\tau}\right)$ as a function of the tau neutrino energy $E_{\nu}$, for the standard 3-active-flavor oscillation framework [1] and in a $3+1$ oscillation scenario, considering a baseline of $L=480 \mathrm{~m}$. Besides results in the standard framework, the upper panel shows results for the $3+1$ scenario with $m_{4}=20 \mathrm{eV}$, for $\left|U_{\tau 4}\right|^{2}=0.08$ and 0.15 , whereas the lower panel shows results for the $3+1$ scenario with $\left|U_{\tau 4}\right|^{2}=0.15$ and $m_{4}=1 \mathrm{eV}, 20 \mathrm{eV}$ and $1 \mathrm{keV}$.

$\mathrm{MeV}$ energy range and below, but not in the energy range of interest (see the position of the peaks of the energy distributions in section 4).

The transition probability in the scenario with three active and one sterile neutrino flavor, in the case when the mass eigenstates fulfill the hierarchy $m_{4} \gg m_{1,2,3}$, can be simplified to [81]

$$
P\left(\nu_{\alpha} \rightarrow \nu_{\beta}\right) \simeq \delta_{\alpha \beta}-4\left(\delta_{\alpha \beta}-\left|U_{\beta n_{\nu}}\right|^{2}\right)\left|U_{\alpha n_{\nu}}\right|^{2} \sin ^{2}\left(\frac{\Delta m^{2} L}{4 E_{\nu}}\right),
$$


where $\Delta m^{2}=m_{4}^{2}-\left(m_{1}^{2}+m_{2}^{2}+m_{3}^{2}\right) / 3 \simeq m_{4}^{2}$. For $m_{4} \sim 20 \mathrm{eV}$, oscillation effects in the tau neutrino survival probability can be pronounced. The highest energy oscillation node with this hierarchy occurs at

$$
E_{\nu-\max [\mathrm{GeV}]}=\frac{\Delta m^{2} L}{2 \pi}=0.807 \Delta m_{[\mathrm{eV}]}^{2} L_{[\mathrm{km}]} .
$$

Introducing a heavy sterile neutrino extends the region of pronounced oscillation dips in the tau neutrino survival probability to a higher energy with examples shown in figure 12 . When $m_{4}=20 \mathrm{eV}$, a survival probability dip occurs at $E_{\nu_{\tau}}=155 \mathrm{GeV}$, an energy near that of the peak of the unoscillated tau neutrino number of events per unit energy. Using eq. (5.2) and $\left|U_{\tau 4}\right|^{2}=0.08$ and 0.15 , the $\nu_{\tau} \rightarrow \nu_{\tau}$ survival probability is shown for $m_{4}=20 \mathrm{eV}$ (upper panel) and $m_{4}=1 \mathrm{eV}, 20 \mathrm{eV}$ and $1 \mathrm{keV}$ (lower panel), all for a baseline of $L=480 \mathrm{~m}$. The values of $\left|U_{\tau 4}\right|^{2}$ are acceptable according to current IceCube constraints [82, 83]. Matter effects can be neglected with the density of the Earth's crust $\rho \approx 2.6 \mathrm{~g} / \mathrm{cm}^{3}$ and the electron fraction $Y_{e} \approx 0.5[84,85]$.

As the lower panel of figure 12 shows, for increasing $m_{4}$ masses, oscillations become rapid over the full neutrino energy range, even at low energies, so their average determines the $\nu_{\tau}$ survival probability. Given the uncertainties in the absolute scale of the $\nu_{\tau}+\bar{\nu}_{\tau}$ flux in the very forward direction, an average decrease in the number of events due to oscillations into sterile neutrinos would be difficult to extract with measurements of forward LHC neutrinos. For this reason, we focus on our example of the case $m_{4}=20 \mathrm{eV}$.

Figure 13 shows the number of events as a function of energy, in the standard threeactive-flavor oscillation framework (black histogram) and in the $3+1$ oscillation framework with $m_{4}=20 \mathrm{eV}$ and $\left|U_{\tau 4}\right|^{2}=0.15$, considering our default heavy-flavor QCD input parameter set and $\left\langle k_{T}\right\rangle=0.7 \mathrm{GeV}$ (left) and $2.2 \mathrm{GeV}$ (right). The orange-dashed histogram shows the effect of $\nu_{\tau}$ disappearance due to oscillations in a $3+1$ scenario with $\left|U_{e 4}\right|^{2}=\left|U_{\mu 4}\right|^{2}=0$, where a dip is visible at neutrino energies $\sim 150 \mathrm{GeV}$.

The oscillation dip may be partially filled in by $\nu_{\mu} \rightarrow \nu_{\tau}$ and $\nu_{e} \rightarrow \nu_{\tau}$ oscillations, where the $\nu_{\mu}$ and $\nu_{e}$ come from heavy-flavor decays and from $\pi^{ \pm}, K^{ \pm}$and $K_{L}$ decays. The NOMAD experiment set the most stringent limits on effective mixing angles with $\nu_{\tau}$ for $3+1$ scenarios with $\Delta m^{2} \simeq m_{4}^{2} \gtrsim 30 \mathrm{eV}^{2}[86,87]$,

$$
\begin{aligned}
& \sin ^{2} 2 \theta_{e \tau} \simeq 4\left|U_{e 4}\right|^{2}\left|U_{\tau 4}\right|^{2}<1.5 \times 10^{-2} \\
& \sin ^{2} 2 \theta_{\mu \tau} \simeq 4\left|U_{\mu 4}\right|^{2}\left|U_{\tau 4}\right|^{2}<3.3 \times 10^{-4} \\
& \sin ^{2} 2 \theta_{e \mu} \simeq 4\left|U_{e 4}\right|^{2}\left|U_{\mu 4}\right|^{2}<1.4 \times 10^{-3} .
\end{aligned}
$$

We use $\left|U_{\tau 4}\right|^{2}=0.15$ to illustrate sterile neutrino mixing effects. ${ }^{5}$ For $\left|U_{\mu 4}\right|^{2}$ and $\left|U_{e 4}\right|^{2}$, eqs. (5.4)-(5.6) must be satisfied. For illustration purposes, we take $\left|U_{\mu 4}\right|^{2}=5 \times 10^{-4}$, a value close to the maximum mixing consistent with eq. (5.5). The Troisk tritium beta decay data can be used to set limits on $\left|U_{e 4}\right|^{2}$ for sterile neutrino masses in the range of $1-100 \mathrm{eV}[89,90]$. For $m_{4}=20 \mathrm{eV},\left|U_{e 4}\right|^{2} \lesssim 6.7 \times 10^{-3}$ comes from the Troisk upper bound. The other constraints, eqs. (5.4) and (5.6), are satisfied by this value of $\left|U_{e 4}\right|^{2}$, considering

\footnotetext{
${ }^{5}$ This is consistent with the $99 \%$ CL limit from ref. [88], where results are shown only for $\Delta m_{41}^{2} \lesssim 10 \mathrm{eV}^{2}$.
} 

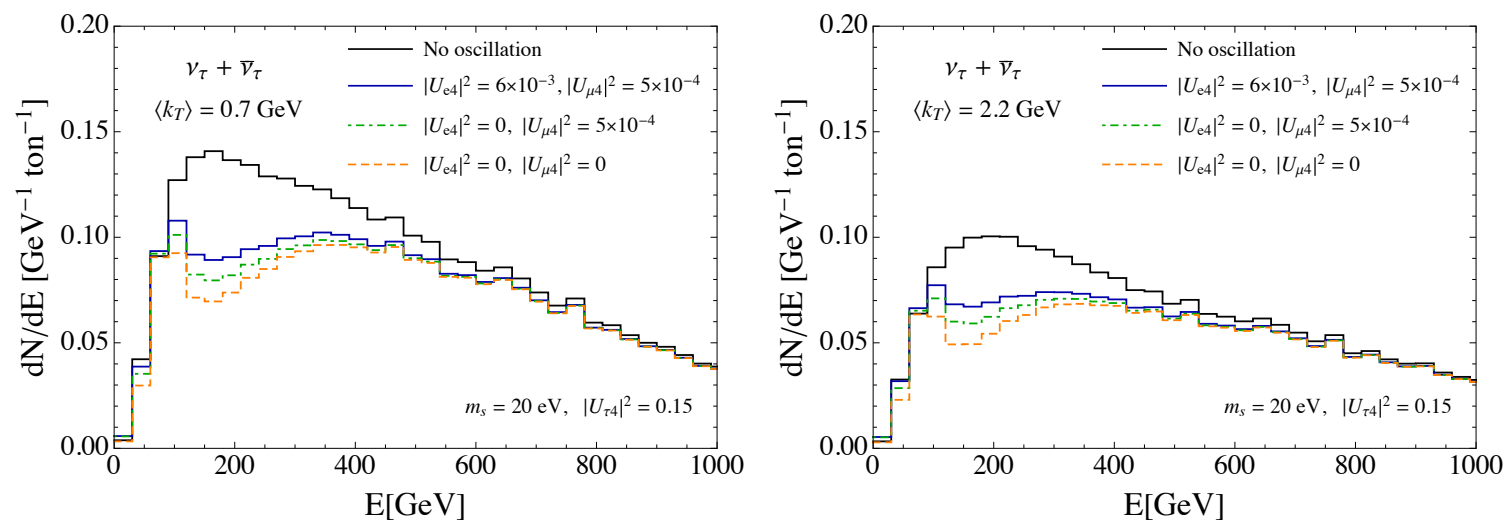

Figure 13. Predictions of the number of $\nu_{\tau}+\bar{\nu}_{\tau}$ charged-current events as a function of neutrino energy in absence of oscillations and in presence of oscillations in a $3+1$ mixing framework, for various choices of the oscillation parameters in the same experimental setup already used in section 4 . Numbers of events are reported for a ton of lead detector, for $\left\langle k_{T}\right\rangle=0.7 \mathrm{GeV}$ (left) and $2.2 \mathrm{GeV}$ (right).

our aforementioned choice for $\left|U_{\tau 4}\right|^{2}$. We use parameters $\left|U_{\mu 4}\right|^{2}=5 \times 10^{-4}$ and a slightly lower value of $\left|U_{e 4}\right|^{2}=6 \times 10^{-3}$, still satisfying the constraints discussed above, in the blue histogram in figure 13. In the figure, $\nu_{e}$ and $\nu_{\mu}$ (and anti-neutrinos) from heavy-flavor decays and from $\pi^{ \pm}, K^{ \pm}$and $K_{L}$ decays are included.

The spectral shape of the number of $\nu_{\tau}+\bar{\nu}_{\tau}$ events changes with different choices of mixing parameters. If $\left|U_{e 4}\right|^{2}=\left|U_{\mu 4}\right|^{2}=0$, spectral distortions will be conspicuous, even if $\left\langle k_{T}\right\rangle=2.2 \mathrm{GeV}$ (corresponding to a total smaller number of events than our default case $\left.\left\langle k_{T}\right\rangle=0.7 \mathrm{GeV}\right)$, as can be seen in the right panel of figure 13 . Keeping the same value of $\left|U_{e 4}^{2}\right|$ and increasing the $\left|U_{\mu 4}\right|^{2}$ value to $\left|U_{\mu 4}\right|^{2}<10^{-3}$, the $\nu_{\tau} \rightarrow \nu_{\tau}$ oscillation dip in the energy distribution of the events is not significantly modified because muon mixing parameters are quite constrained as discussed above. This effect is shown in the green histogram of figure 13, obtained by setting $\left|U_{e 4}\right|^{2}=0$ and $\left|U_{\mu 4}\right|^{2}=5 \times 10^{-4}$. When $\left|U_{e 4}\right|^{2} \neq 0$, electron neutrinos coming primarily from $K_{e 3}^{0}$ that oscillate to $\nu_{\tau}$ because of sterile neutrino mixing additionally fill in the spectral distortion of the $\nu_{\tau}+\bar{\nu}_{\tau}$ event number as shown in figure 13 with the solid blue histogram for $\left|U_{e 4}\right|^{2}=6 \times 10^{-3}$.

Untangling the physics of a $3+1$ flavor oscillation scenario, from the standard 3-flavor oscillation scenario, considering the QCD theoretical uncertainties related to the choice of the scales and of the phenomenological $\left\langle k_{T}\right\rangle$ smearing parameter, will be difficult if the mixing parameters $\left|U_{e 4}\right|^{2}$ and $\left|U_{\mu 4}\right|^{2}$ are close to $6 \times 10^{-3}$ and $5 \times 10^{-4}$, respectively. Although the values of $\left|U_{e 4}\right|^{2}$ and $\left|U_{\mu 4}\right|^{2}$ are small, the large number of $\nu_{e}$ and $\nu_{\mu}$ from light-meson production and decay means that $\nu_{e}$ and $\nu_{\mu}$ mixing with sterile neutrinos can have a non-negligible impact. The challenge is illustrated in figures 14 and 15 . These figures show the numbers of tau neutrino plus antineutrino charged-current events, with each distribution normalized to the corresponding number of events at $1 \mathrm{TeV}$ for that distribution, to highlight the effect of the scale dependence and of various values of $\left\langle k_{T}\right\rangle$ on the shape of the distribution. In figure $14,\left\langle k_{T}\right\rangle=0.7 \mathrm{GeV}$ is fixed and the scales are varied. In particular, although a peak is still visible, the case with all three elements $\left|U_{\ell 4}\right|^{2} \neq 0$ in 

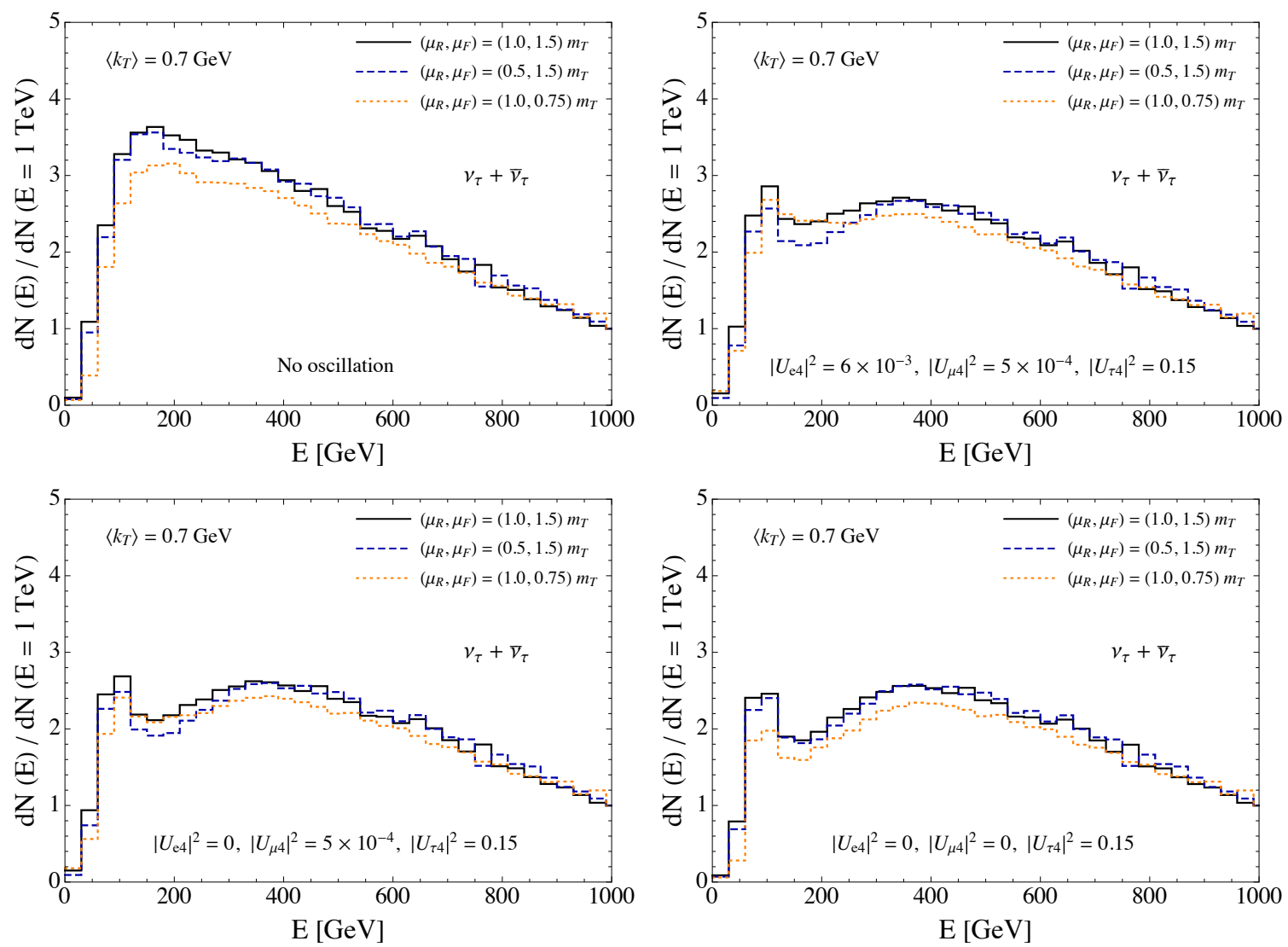

Figure 14. The ratio of the number of $\nu_{\tau}+\bar{\nu}_{\tau}$ charged-current interaction events and the number of events at $1 \mathrm{TeV}$ in each case, as a function of neutrino energy, without oscillations (upper left plot) and in a $3+1$ oscillation scenarios with different mixing parameters. The numbers of events are evaluated for $\left\langle k_{T}\right\rangle=0.7 \mathrm{GeV}$ for three different $\left(\mu_{R}, \mu_{F}\right)$ scale choices that bracket the scale uncertainties.

the upper right panel presents a slightly shallower dip in the oscillated $3+1$ spectrum as compared to the case with $\left|U_{e 4}\right|^{2}=0$ and $\left|U_{\mu 4}\right|^{2}=0$ shown in the lower right panel. The normalization of each distribution in figure 14 to its own distribution at $E=1 \mathrm{TeV}$ collapses the scale uncertainty band of figure 6 . Figure 15 shows that a large transverse momentum smearing can somewhat obscure the spectral features of $3+1$ oscillations as well. The effects of $\nu_{e}$ and $\nu_{\mu}$ mixing with sterile neutrinos are more important in each panel of figures 14 and 15 , namely, for $\left(\mu_{R}, \mu_{F}\right)=(1.0,0.75) m_{T}$ in figure 14 and $\left\langle k_{T}\right\rangle=2.2 \mathrm{GeV}$ in figure 15 . The dip in the spectrum is least pronounced in the case of $\left(\mu_{R}, \mu_{F}\right)=(1.0,0.75) m_{T}$ and $\left\langle k_{T}\right\rangle=2.2 \mathrm{GeV}$. A dedicated study of $3+1$ oscillation scenarios that includes detector resolution effects would be important to understand in detail the reach of a forward tau neutrino experiment along the LHC beamline. 

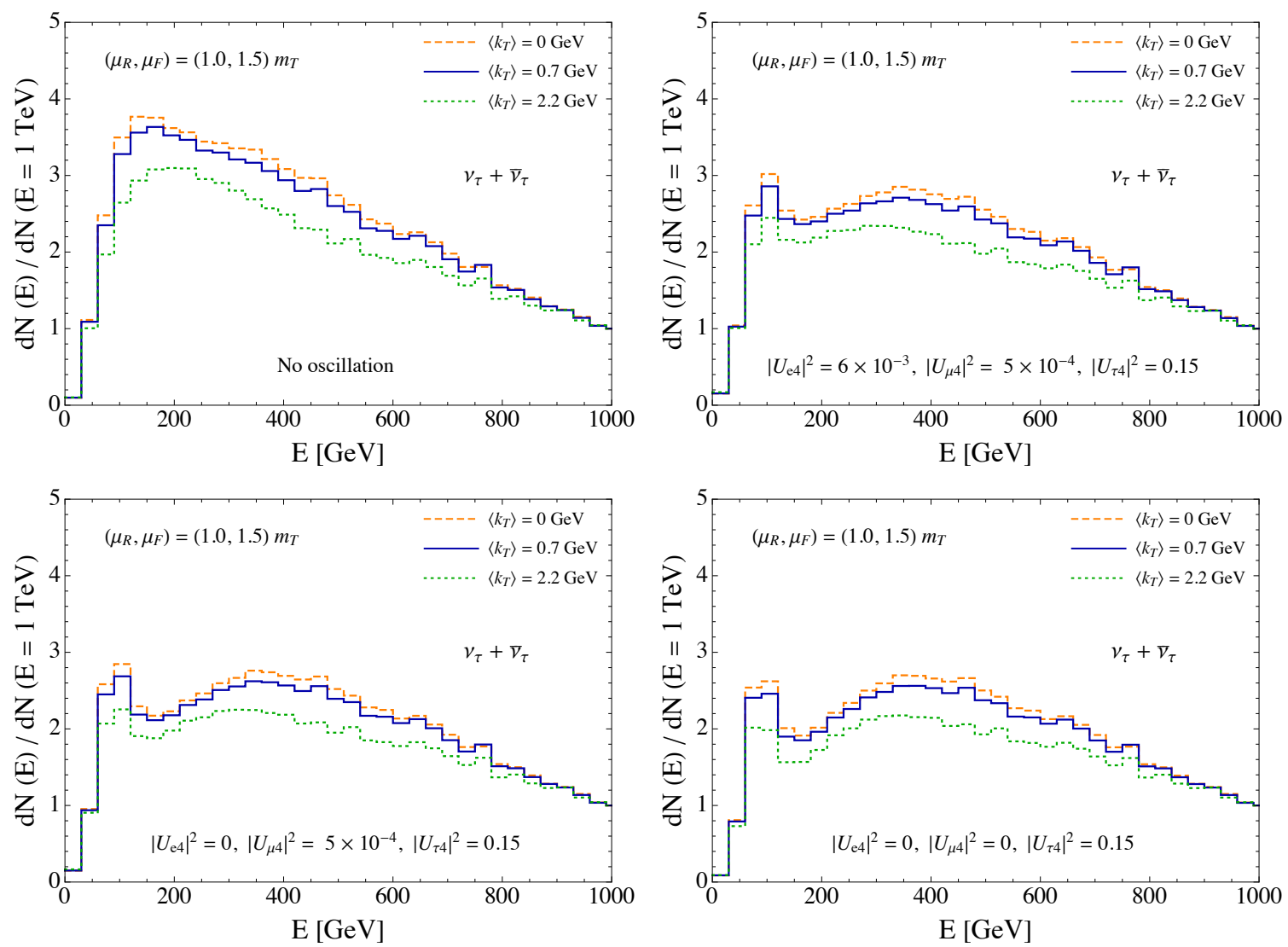

Figure 15. The same as figure 14, but considering different values of $\left\langle k_{T}\right\rangle$, for our default $\left(\mu_{R}\right.$, $\left.\mu_{F}\right)$ scale choice.

\section{Conclusions}

Theoretical proposals to exploit collider production of tau neutrinos and antineutrinos via heavy-flavor decays in the far-forward region have a long history. Recent proposals of experiments to detect BSM particles with feeble interactions have made our evaluation timely. This work provides a first evaluation of the number of $\nu_{\tau}+\bar{\nu}_{\tau}$ charged-current interaction events in the very forward region, including QCD effects beyond the leading order/leading logarithmic accuracy considered in previous estimates and studying the effects of different sources of QCD uncertainties previously neglected. We focus on neutrinos with pseudorapidities $\eta>6.87$, consistently with the geometry of a $1 \mathrm{~m}$ radius cylindrical detector at a distance of $480 \mathrm{~m}$ from the interaction point [6-8] to illustrate a number of effects.

Thousands of tau neutrino plus antineutrino events are predicted for 1 meter of lead (35.6 ton) target. Theoretical uncertainties are quite large due to, in particular, the renormalization and factorization scale variation in the heavy-flavour production cross-sections. We show results for both $\left(\mu_{R}, \mu_{F}\right)=(1.0,1.5) m_{T}$ and $\left(\mu_{R}, \mu_{F}\right)=(1.0,1.0) m_{T}$, to better match the LHCb data and for comparisons with the usual scale conventions adopted in QCD studies of heavy-flavor production, respectively. Seven-point scale variation around the central scale combinations $\left(\mu_{R}, \mu_{F}\right)=(1.0,1.0) m_{T}$ and $(1.0,1.5) m_{T}$ causes differences 
amounting to a factor of $\sim 4-6$ between the upper and lower limit of the predicted event numbers, for the intrinsic momentum parameter with which our NLO predictions convoluted with fragmentation functions approximately reproduce PowHEG + PYTHIA results.

The introduction of the parameter $\left\langle k_{T}\right\rangle$ in the Gaussian smearing factor $f\left(\vec{k}_{T}\right)$ in eq. (3.1) distorts the far-forward tau neutrino and antineutrino spectra. This is also the case in fixed-target experiments like SHiP [62]. The collinear parton model is sufficient for central collisions, but for forward production, as we have shown, non-collinear effects have a significant impact on the number of events for forward neutrino production at the LHC. Although the Gaussian form of $f\left(\vec{k}_{T}\right)$ is imperfect, it can approximate non-perturbative QCD effects and mimic part of the perturbative effects beyond fixed-order. A comparison with LHCb double-differential cross sections in $p_{T}$ and rapidity for $D_{s}$ production in $p p$ collisions at $\sqrt{s}=13 \mathrm{TeV}$, for $p_{T} \in[0,14] \mathrm{GeV}$ in five rapidity bins in the range $y=2.0$ 4.5 , shows that the experimental data are reasonably reproduced by theoretical predictions in a framework combining NLO pQCD corrections to the hard-scattering, $k_{T}$-smearing effects and phenomenological fragmentation functions. Experiments that probe heavyflavor physics in the very forward region (e.g., ref. [91]) will help to better quantify and disentangle the need of accurate procedures jointly resumming different kinds of logarithms and that of more rigorous descriptions of non-perturbative QCD effects, as well as the limits of the collinear approximation. The inclusion of both small $x$ and $k_{T}$ effects in theoretical evaluations of heavy-flavor production at the Electron-Ion Collider (EIC) have implications for measurements (see, e.g., refs. [92, 93]). In another arena, predictions of the prompt atmospheric neutrino flux $[49,50,61,94-96]$ will also be constrained by measurements of the tau neutrino energy spectrum in the far-forward region at the LHC.

For a baseline of $\sim 500 \mathrm{~m}$, oscillations in the standard 3-flavor scenario are negligible at the energy scales of the tau neutrino beams in our setup. On the other hand, in a $3+1$ oscillation scenario, including a sterile neutrino in the $\mathcal{O}(20 \mathrm{eV})$ mass range with three active flavor eigenstates, oscillations could give rise to visible signals in the energy dependence of the tau neutrino events. The contribution to $\nu_{\tau}$ events due to $\nu_{\mu} \rightarrow \nu_{\tau}$ oscillations is not important due to the small value of $\left|U_{\mu 4}\right|^{2}$. This is the case even though there are $\sim 100$ times more $\nu_{\mu}+\bar{\nu}_{\mu}$ produced from charged pion and kaon decays than from heavy-flavor decays. The number of $\nu_{\mu}+\bar{\nu}_{\mu}$ from heavy flavor decays is larger than the number of $\nu_{\tau}+\bar{\nu}_{\tau}$ by a factor of $\sim 10$. The $\nu_{e} \rightarrow \nu_{\tau}$ oscillation effects are also small.

Therefore a large number of $\nu_{\tau}+\bar{\nu}_{\tau}$ events in this energy range would provide an opportunity to constrain $3+1$ oscillation models with a sterile neutrino in the 10 's of eV mass range. In the case of $\nu_{\tau}$ disappearance, the location of a dip in the charged-current event distribution as a function of tau neutrino energy will constrain the mass of the fourth mass eigenstate (mostly sterile neutrino) $m_{4}$. The quantity $\left|U_{\tau 4}\right|^{2}$ is currently poorly constrained. We showed that, as long as $\nu_{e} \rightarrow \nu_{\tau}$ appearance is suppressed, for $\left|U_{\tau 4}\right|^{2}=0.15$ in principle the oscillation effect would be unambiguous. However, uncertainties in heavyflavor production present challenges to precision constraints on the $3+1$ sterile neutrino parameter space accessible to a far-forward neutrino experiment at the LHC. Practical aspects of tau neutrino detection in the high-luminosity environment will also be a challenge because of the muon neutrino background. 


\section{A Decay distributions}

The two-body decays of the $D_{s}$ meson in its rest frame come from energy and momentum conservation. Keeping the polarization of the $\tau$, the energy and angular distribution of the tau neutrino can also be obtained [59, 97, 98]. Detailed formulas for the direct $\nu_{\tau}$ and chain decay $\nu_{\tau}$ distributions appear in appendix B of ref. [62]. Decays in the $D_{s}$ and $\tau$ rest frames are appropriatedly boosted to the collider frame, including full angular dependence.

Neutrinos can be produced through the three-body semileptonic decay process of $D$ and $B$ mesons, $D(B) \rightarrow K(D) l \nu_{l}(l=e, \mu)$. The distribution of neutrinos from the decay in the rest frame is respectively given by

$$
\frac{\mathrm{d} \Gamma\left(h_{i} \rightarrow \nu_{l}\right)}{\mathrm{d} x_{\nu}} \sim \begin{cases}\frac{m_{D}^{5} x_{\nu}^{2}\left(1-r_{h}-x_{\nu}\right)^{2}\left[3+r_{h}\left(3-x_{\nu}\right)-5 x_{\nu}+2 x_{\nu}^{2}\right]}{\left(1-x_{\nu}\right)^{3}} & \text { for } \quad h_{i}=D \\ \frac{m_{B}^{5} x_{\nu}^{2}\left(1-r_{h}-x_{\nu}\right)^{2}}{\left(1-x_{\nu}\right)} & \text { for } \quad h_{i}=B\end{cases}
$$

where the fraction of energy transferred to the neutrino is $x_{\nu}=2 E_{\nu} / m_{i}$ and the hadron mass fraction is $r_{h}=m_{f}^{2} / m_{i}^{2}$, with $m_{i}$ and $m_{f}$ being the hadron masses in the initial and final states, respectively. The corresponding cumulative distribution functions (CDF) are

$$
\begin{aligned}
& F\left(x_{\nu}\right)=\frac{1}{\Gamma} \int_{0}^{x_{\nu}} d x^{\prime} \frac{\mathrm{d} \Gamma}{\mathrm{d} x^{\prime}} \\
& {[1 \mathrm{em}]=\left\{\begin{array}{r}
\frac{1}{D\left(r_{h}\right)\left(1-x_{\nu}\right)^{2}}\left[x _ { \nu } \left(2 r_{h}^{3} x_{\nu}^{2}-6 r_{h}^{2}\left(2-x_{\nu}\right)\left(1-x_{\nu}\right)+\left(2-x_{\nu}\right)\left(1-x_{\nu}\right)^{2} x_{\nu}^{2}\right.\right. \\
\left.\left.-2 r_{h}\left(1-x_{\nu}\right)^{2} x_{\nu}^{2}\right)-12 r_{h}^{2}\left(1-x_{\nu}\right)^{2} \ln \left[1-x_{\nu}\right]\right] \quad \text { for } \quad h_{i}=D \\
\frac{1}{D\left(r_{h}\right)}\left[x_{\nu}\left(x_{\nu}^{2}\left(4-3 x_{\nu}\right)-8 r_{h} x_{\nu}^{2}-6 r_{h}^{2}\left(2+x_{\nu}\right)\right)-12 r_{h}^{2} \ln \left[1-x_{\nu}\right]\right] \quad \text { for } \quad h_{i}=B
\end{array}\right.}
\end{aligned}
$$

where $D(r)=1-8 r-12 r^{2} \ln (r)+8 r^{3}-r^{4}$. The CDF is used to determine the neutrino energy in the heavy meson rest frame, and along with an isotropic decay distribution, the neutrino four-momentum in the rest frame is boosted to the collider frame, where the heavy meson $D(B)$ has four-momentum $p_{D(B)}$.

The $B$ meson also decays to tau neutrinos via $B \rightarrow D \tau \nu_{\tau}$, and the $\tau$ lepton decays subsequently to $\nu_{\tau}$. In this case, there is an additional effect due to the non-negligible mass of $\tau$. With the additional mass term $r_{\tau}=m_{\tau}^{2} / m_{B}^{2}$, one obtains the distributions of $\tau$ and $\nu_{\tau}$ from $B$ decays:

$$
\begin{aligned}
\frac{\mathrm{d} \Gamma(B \rightarrow \tau)}{\mathrm{d} x_{\tau}} \sim & \frac{m_{B}^{5}}{\left(1+r_{\tau}-x_{\tau}\right)^{3}}\left(1-x_{\tau}+r_{\tau}-r_{h}\right)^{2}\left(x_{\tau}^{2}-4 r_{\tau}\right)^{1 / 2}\left[r_{\tau}^{2}\left(3 x_{\tau}-4\right)\right. \\
& \left.+x\left(3+2 x_{\tau}^{2}-5 x_{\tau}+r_{h}\left(3-x_{\tau}\right)\right)-r_{\tau}\left(4+5 x_{\tau}^{2}-10 x_{\tau}+r_{h}\left(8-3 x_{\tau}\right)\right)\right], \\
\frac{\mathrm{d} \Gamma\left(B \rightarrow \nu_{\tau}\right)}{\mathrm{d} x_{\nu}} \sim & \frac{m_{B}^{5}}{1-x_{\nu}} x_{\nu}^{2}\left(1-r_{h}-r_{\tau}-x_{\nu}\right)\left(r_{h}^{2}-2 r_{h}\left(1+r_{\tau}-x_{\nu}\right)+\left(1-r_{\tau}-x_{\nu}\right)^{2}\right)^{1 / 2} .
\end{aligned}
$$



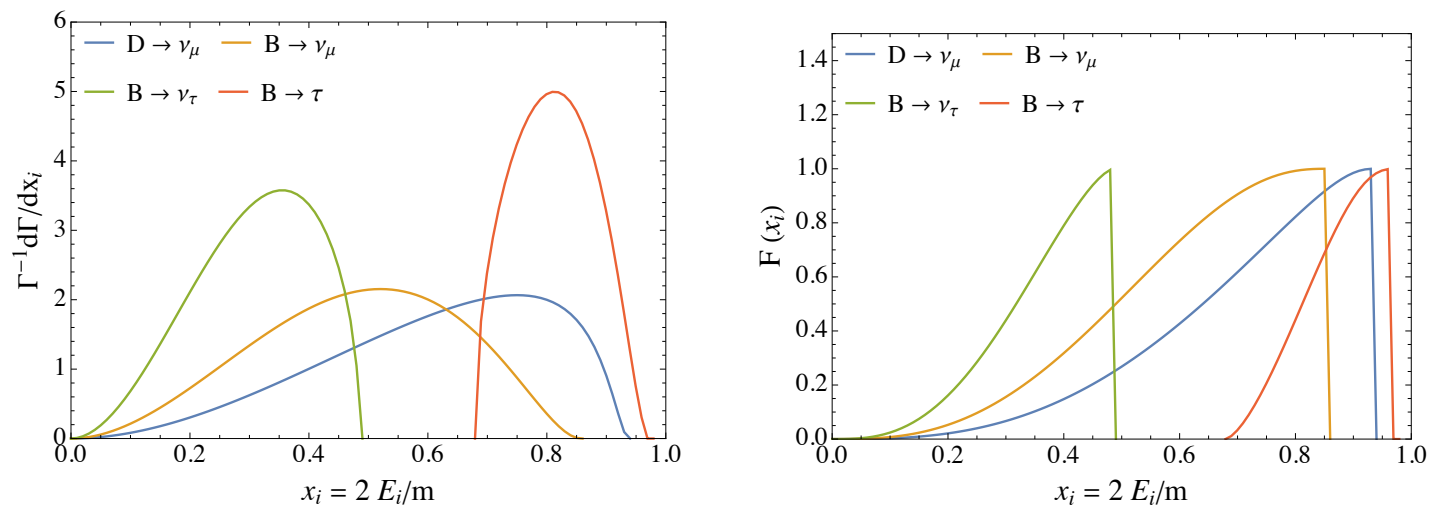

Figure 16. Left panel: the fractional energy distributions $x_{i}=E_{i} /(m / 2)$ in three-body semileptonic decays in the rest frame of the decaying heavy particle with $m=m_{D_{s}}$ and $m=m_{B}$ and $i=\nu_{\mu}, \nu_{\tau}$ and $\tau$. Right panel: the cumulative distribution function for each of the three-body decays shown in the left panel.

Here, $x_{\tau}=2 E_{\tau} / m_{i}$ is the fraction of energy transferred to tau. The expression of the CDF for $\tau$ and $\nu_{\tau}$ from $B$ decays is too complicated to be presented here. Again, both energy and angular distributions are determined in the rest frame, then boosted to the collider frame. For reference, we show the fractional energy distribution (left) and CDFs (right) for $\nu_{\mu}, \nu_{\tau}$ and $\tau$ in the rest frame of the decaying $D$ and $B$ in figure 16 for the three-body decays.

The energy fractions $x$ span the following range:

$$
\begin{aligned}
0 & <x_{\nu_{l}}<1-\left(\sqrt{r_{l}}+\sqrt{r_{h}}\right)^{2} \\
2 \sqrt{r_{\tau}} & <x_{\tau}<1+r_{\tau}-r_{h} .
\end{aligned}
$$

For electron neutrino $\left(\nu_{e}\right)$ and muon neutrino $\left(\nu_{\mu}\right)$, the maximum value of $x_{\nu}$ is simply $x_{\nu}^{\max }=1-r_{h}$.

\section{Acknowledgments}

This work is supported in part by Department of Energy grants DE-SC-0010113, DE-SC0012704, and the Korean Research Foundation (KRF) through the CERN-Korea Fellowship program. The authors would like to express a special thanks to the Mainz Institute for Theoretical Physics (MITP) of the Cluster of Excellence PRISMA+ (Project ID 39083149) for its hospitality and support. We thank the Rencontres du Vietnam/VietNus 2017 workshop at the International Center for Interdisciplinary Science and Education (ICISE), QuiNhon, Vietnam for support and discussions in the early stage of this work, and we thank C. Giunti and M.L. Mangano for comments and discussions. M.V.G. is grateful to the II Institute for Theoretical Physics of the University of Hamburg for hospitality during the completion of this work. 
Open Access. This article is distributed under the terms of the Creative Commons Attribution License (CC-BY 4.0), which permits any use, distribution and reproduction in any medium, provided the original author(s) and source are credited.

\section{References}

[1] Particle Data Group collaboration, Review of Particle Physics, Chin. Phys. C 40 (2016) 100001 [INSPIRE].

[2] DUNE collaboration, Long-Baseline Neutrino Facility (LBNF) and Deep Underground Neutrino Experiment (DUNE), arXiv:1512.06148 [INSPIRE].

[3] DUNE collaboration, Long-Baseline Neutrino Facility (LBNF) and Deep Underground Neutrino Experiment (DUNE), arXiv:1601.05471 [INSPIRE].

[4] DUNE collaboration, The DUNE Far Detector Interim Design Report Volume 1: Physics, Technology and Strategies, arXiv:1807.10334 [INSPIRE].

[5] H. Park, The estimation of neutrino fluxes produced by proton-proton collisions at $\sqrt{s}=14 \mathrm{TeV}$ of the LHC, JHEP 10 (2011) 092 [arXiv:1110.1971] [INSPIRE].

[6] J.L. Feng, I. Galon, F. Kling and S. Trojanowski, ForwArd Search ExpeRiment at the LHC, Phys. Rev. D 97 (2018) 035001 [arXiv:1708.09389] [INSPIRE].

[7] FASER collaboration, Technical Proposal for FASER: ForwArd Search ExpeRiment at the $L H C$, arXiv: 1812.09139 [INSPIRE].

[8] FASER collaboration, FASER: ForwArd Search ExpeRiment at the LHC, arXiv: 1901.04468 [INSPIRE].

[9] S. Buontempo, G.M. Dallavalle, G. De Lellis, D. Lazic and F.L. Navarria, CMS-XSEN: LHC Neutrinos at CMS. Experiment Feasibility Study, arXiv:1804.04413 [INSPIRE].

[10] N. Beni et al., Physics Potential of an Experiment using LHC Neutrinos, J. Phys. G 46 (2019) 115008 [arXiv:1903.06564] [INSPIRE].

[11] XSEN collaboration, XSEN: a $\nu N$ Cross section Measurement using High Energy Neutrinos from pp collisions at the LHC, arXiv: 1910.11340 [INSPIRE].

[12] A. De Rujula and R. Ruckl, Neutrino and muon physics in the collider mode of future accelerators, in proceedings of ECFA-CERN Workshop on large hadron collider in the LEP tunnel, Lausanne and CERN, Geneva, Switzerland, 21-27 March 1984, vol. 2, pp. 571-596 (1984) [DOI].

[13] K. Winter, Detection of the tau-neutrino at the LHC, in proceedings of ECFA Large Hadron Collider Workshop, Aachen, Germany, 4-9 October 1990, vol. 2, pp. 37-49 (1990)[InSPIRE].

[14] A. De Rujula, E. Fernandez and J.J. Gomez-Cadenas, Neutrino fluxes at future hadron colliders, Nucl. Phys. B 405 (1993) 80 [inSPIRE].

[15] F. Vannucci, Neutrino physics at LHC/SSC, in 5th International Symposium on Neutrino Telescopes, Venice, Italy, 2-4 March 1993, pp. 57-68 (1993) [INSPIRE].

[16] LHCb collaboration, Measurement of $D_{s}^{ \pm}$production asymmetry in pp collisions at $\sqrt{s}=7$ and $8 \mathrm{TeV}$, JHEP 08 (2018) 008 [arXiv: 1805.09869] [INSPIRE].

[17] T. Sjöstrand, The PYTHIA Event Generator: Past, Present and Future, Comput. Phys. Commun. 246 (2020) 106910 [arXiv: 1907.09874] [INSPIRE]. 
[18] FASER collaboration, Detecting and Studying High-Energy Collider Neutrinos with FASER at the LHC, Eur. Phys. J. C 80 (2020) 61 [arXiv:1908.02310] [INSPIRE].

[19] FASER collaboration, Technical Proposal: FASERnu, arXiv:2001.03073 [INSPIRE].

[20] T. Sjöstrand et al., An Introduction to PYTHIA 8.2, Comput. Phys. Commun. 191 (2015) 159 [arXiv:1410.3012] [INSPIRE].

[21] SHIP collaboration, SND@LHC, arXiv:2002.08722 [INSPIRE].

[22] DONUT collaboration, Observation of tau neutrino interactions, Phys. Lett. B 504 (2001) 218 [hep-ex/0012035] [INSPIRE].

[23] DONuT collaboration, Final tau-neutrino results from the DONuT experiment, Phys. Rev. D 78 (2008) 052002 [arXiv:0711.0728] [INSPIRE].

[24] OPERA collaboration, Recent results of the OPERA experiment, AIP Conf. Proc. 1743 (2016) 060004 [INSPIRE].

[25] OPERA collaboration, Final Results of the OPERA Experiment on $\nu_{\tau}$ Appearance in the CNGS Neutrino Beam, Phys. Rev. Lett. 120 (2018) 211801 [Erratum ibid. 121 (2018) 139901] [arXiv: 1804.04912] [INSPIRE].

[26] Super-Kamiokande collaboration, Measurement of the tau neutrino cross section in atmospheric neutrino oscillations with Super-Kamiokande, Phys. Rev. D 98 (2018) 052006 [arXiv: 1711.09436] [INSPIRE].

[27] IceCube collaboration, Measurement of Atmospheric Tau Neutrino Appearance with IceCube DeepCore, Phys. Rev. D 99 (2019) 032007 [arXiv: 1901.05366] [INSPIRE].

[28] ICECUBE collaboration, Measurement of the multi-TeV neutrino cross section with IceCube using Earth absorption, Nature 551 (2017) 596 [arXiv:1711.08119] [INSPIRE].

[29] M. Bustamante and A. Connolly, Extracting the Energy-Dependent Neutrino-Nucleon Cross section above $10 \mathrm{TeV}$ Using IceCube Showers, Phys. Rev. Lett. 122 (2019) 041101 [arXiv: 1711.11043] [INSPIRE].

[30] P. Nason, S. Dawson and R.K. Ellis, The Total Cross-Section for the Production of Heavy Quarks in Hadronic Collisions, Nucl. Phys. B 303 (1988) 607 [INSPIRE].

[31] P. Nason, S. Dawson and R.K. Ellis, The One Particle Inclusive Differential Cross-Section for Heavy Quark Production in Hadronic Collisions, Nucl. Phys. B 327 (1989) 49 [Erratum ibid. B 335 (1990) 260] [INSPIRE].

[32] M.L. Mangano, P. Nason and G. Ridolfi, Heavy quark correlations in hadron collisions at next-to-leading order, Nucl. Phys. B 373 (1992) 295 [INSPIRE].

[33] LHCb collaboration, Measurements of prompt charm production cross-sections in pp collisions at $\sqrt{s}=13 \mathrm{TeV}$, JHEP 03 (2016) 159 [Erratum ibid. 09 (2016) 013] [arXiv: 1510.01707] [INSPIRE].

[34] H.B.J. Koers, A. Pe'er and R.A. M.J. Wijers, Parameterization of the energy and rapidity distributions of secondary pions and kaons produced in energetic proton-proton collisions, hep-ph/0611219 [INSPIRE].

[35] O.S. Bruning et al., LHC Design Report Vol. 1: The LHC Main Ring, CERN-2004-003-V1 (2004) [INSPIRE].

[36] L. Apanasevich et al., $k_{T}$ effects in direct photon production, Phys. Rev. D 59 (1999) 074007 [hep-ph/9808467] [INSPIRE]. 
[37] M.L. Mangano, Two lectures on heavy quark production in hadronic collisions, Proc. Int. Sch. Phys. Fermi 137 (1998) 95 [hep-ph/9711337] [InSPIRE].

[38] G. Miu and T. Sjöstrand, W production in an improved parton shower approach, Phys. Lett. B 449 (1999) 313 [hep-ph/9812455] [INSPIRE].

[39] C. Balázs, J. Huston and I. Puljak, Higgs production: A Comparison of parton showers and resummation, Phys. Rev. D 63 (2001) 014021 [hep-ph/0002032] [INSPIRE].

[40] P.Z. Skands, Tuning Monte Carlo Generators: The Perugia Tunes, Phys. Rev. D 82 (2010) 074018 [arXiv: 1005. 3457] [INSPIRE].

[41] S. Catani, M. Ciafaloni and F. Hautmann, High-energy factorization and small $x$ heavy flavor production, Nucl. Phys. B 366 (1991) 135 [InSPIRE].

[42] J.C. Collins and R.K. Ellis, Heavy quark production in very high-energy hadron collisions, Nucl. Phys. B 360 (1991) 3 [INSPIRE].

[43] C. Peterson, D. Schlatter, I. Schmitt and P.M. Zerwas, Scaling Violations in Inclusive $e^{+} e^{-}$ Annihilation Spectra, Phys. Rev. D 27 (1983) 105 [InSPIRE].

[44] M. Lisovyi, A. Verbytskyi and O. Zenaiev, Combined analysis of charm-quark fragmentation-fraction measurements, Eur. Phys. J. C 76 (2016) 397 [arXiv:1509.01061] [INSPIRE].

[45] Particle Data Group collaboration, Review of Particle Physics, Phys. Rev. D 98 (2018) 030001 [INSPIRE].

[46] LHCb collaboration, Measurement of b hadron fractions in $13 \mathrm{TeV}$ pp collisions, Phys. Rev. D 100 (2019) 031102 [arXiv: 1902.06794] [INSPIRE].

[47] K. Kovarik et al., nCTEQ15 - Global analysis of nuclear parton distributions with uncertainties in the CTEQ framework, Phys. Rev. D 93 (2016) 085037 [arXiv:1509.00792] [INSPIRE].

[48] M. Cacciari, S. Frixione, N. Houdeau, M.L. Mangano, P. Nason and G. Ridolfi, Theoretical predictions for charm and bottom production at the LHC, JHEP 10 (2012) 137 [arXiv: 1205.6344] [INSPIRE].

[49] M. Benzke, M.V. Garzelli, B. Kniehl, G. Kramer, S. Moch and G. Sigl, Prompt neutrinos from atmospheric charm in the general-mass variable-flavor-number scheme, JHEP 12 (2017) 021 [arXiv: 1705.10386] [INSPIRE].

[50] PROSA collaboration, Improved constraints on parton distributions using LHCb, ALICE and HERA heavy-flavour measurements and implications for the predictions for prompt atmospheric-neutrino fluxes, JHEP 04 (2020) 118 [arXiv:1911.13164] [INSPIRE].

[51] LHCb collaboration, Measurement of the $B^{ \pm}$production cross-section in pp collisions at $\sqrt{s}=7$ and $13 \mathrm{TeV}$, JHEP 12 (2017) 026 [arXiv:1710.04921] [INSPIRE].

[52] W. Bai, M. Diwan, M.V. Garzelli, Y.S. Jeong and M.H. Reno, in preparation.

[53] U. D'Alesio, F. Murgia, C. Pisano and P. Taels, Probing the gluon Sivers function in $p^{\uparrow} p \rightarrow J / \psi X$ and $p^{\uparrow} p \rightarrow D X$, Phys. Rev. D 96 (2017) 036011 [arXiv: 1705. 04169] [INSPIRE].

[54] LHCb collaboration, Measurement of the $J / \psi$ pair production cross-section in pp collisions at $\sqrt{s}=13 \mathrm{TeV}$, JHEP 06 (2017) 047 [Erratum ibid. 10 (2017) 068] [arXiv:1612.07451] [INSPIRE]. 
[55] J.-P. Lansberg, C. Pisano, F. Scarpa and M. Schlegel, Pinning down the linearly-polarised gluons inside unpolarised protons using quarkonium-pair production at the LHC, Phys. Lett. B 784 (2018) 217 [Erratum ibid. B 791 (2019) 420] [arXiv:1710.01684] [INSPIRE].

[56] A. Bacchetta, G. Bozzi, M. Radici, M. Ritzmann and A. Signori, Effect of Flavor-Dependent Partonic Transverse Momentum on the Determination of the $W$ Boson Mass in Hadronic Collisions, Phys. Lett. B 788 (2019) 542 [arXiv:1807.02101] [InSPIRE].

[57] S. Frixione, P. Nason and C. Oleari, Matching NLO QCD computations with Parton Shower simulations: the POWHEG method, JHEP 11 (2007) 070 [arXiv: 0709. 2092] [INSPIRE].

[58] S. Frixione, P. Nason and G. Ridolfi, A Positive-weight next-to-leading-order Monte Carlo for heavy flavour hadroproduction, JHEP 09 (2007) 126 [arXiv:0707.3088] [INSPIRE].

[59] S.M. Barr, T.K. Gaisser, P. Lipari and S. Tilav, Ratio of $\nu_{e} / \nu_{\mu}$ in Atmospheric Neutrinos, Phys. Lett. B 214 (1988) 147 [inSPIRE].

[60] L. Pasquali and M.H. Reno, Tau-neutrino fluxes from atmospheric charm, Phys. Rev. D 59 (1999) 093003 [hep-ph/9811268] [INSPIRE].

[61] A. Bhattacharya et al., Prompt atmospheric neutrino fluxes: perturbative QCD models and nuclear effects, JHEP 11 (2016) 167 [arXiv:1607.00193] [INSPIRE].

[62] W. Bai and M.H. Reno, Prompt neutrinos and intrinsic charm at SHiP, JHEP 02 (2019) 077 [arXiv: 1807.02746] [INSPIRE].

[63] S. Kretzer and M.H. Reno, Tau neutrino deep inelastic charged current interactions, Phys. Rev. D 66 (2002) 113007 [hep-ph/0208187] [INSPIRE].

[64] S. Kretzer and M.H. Reno, Target mass corrections to electroweak structure functions and perturbative neutrino cross-sections, Phys. Rev. D 69 (2004) 034002 [hep-ph/0307023] [INSPIRE].

[65] Y.S. Jeong and M.H. Reno, Quark mass effects in high energy neutrino nucleon scattering, Phys. Rev. D 81 (2010) 114012 [arXiv:1001.4175] [INSPIRE].

[66] Y.S. Jeong and M.H. Reno, Tau neutrino and antineutrino cross sections, Phys. Rev. D 82 (2010) 033010 [arXiv: 1007.1966] [INSPIRE].

[67] M.H. Reno, Electromagnetic structure functions and neutrino nucleon scattering, Phys. Rev. D 74 (2006) 033001 [hep-ph/0605295] [INSPIRE].

[68] P. Lipari, M. Lusignoli and F. Sartogo, The Neutrino cross-section and upward going muons, Phys. Rev. Lett. 74 (1995) 4384 [hep-ph/9411341] [INSPIRE].

[69] S. Kretzer and M.H. Reno, sigma DIS (nu N), NLO perturbative QCD and $O(1 \mathrm{GeV}$ ) mass corrections, Nucl. Phys. Proc. Suppl. 139 (2005) 134 [hep-ph/0410184] [INSPIRE].

[70] Cosmic Ray Monte Carlo package, https://web.ikp.kit.edu/rulrich/crmc.html.

[71] T. Pierog, I. Karpenko, J.M. Katzy, E. Yatsenko and K. Werner, EPOS LHC: Test of collective hadronization with data measured at the CERN Large Hadron Collider, Phys. Rev. C 92 (2015) 034906 [arXiv:1306.0121] [INSPIRE].

[72] S. Ostapchenko, Monte Carlo treatment of hadronic interactions in enhanced Pomeron scheme: I. QGSJET-II model, Phys. Rev. D 83 (2011) 014018 [arXiv:1010.1869] [INSPIRE].

[73] F. Riehn, R. Engel, A. Fedynitch, T.K. Gaisser and T. Stanev, The hadronic interaction model Sibyll 2.3c and extensive air showers, arXiv:1912.03300 [INSPIRE]. 
[74] A. Fedynitch, F. Riehn, R. Engel, T.K. Gaisser and T. Stanev, Hadronic interaction model sibyll 2.3c and inclusive lepton fluxes, Phys. Rev. D 100 (2019) 103018 [arXiv:1806.04140] [INSPIRE].

[75] F. Riehn, H.P. Dembinski, R. Engel, A. Fedynitch, T.K. Gaisser and T. Stanev, The hadronic interaction model SIBYLL 2.3c and Feynman scaling, PoS(ICRC2017) 301 (2018) [arXiv: 1709.07227] [INSPIRE].

[76] V. Cirigliano, M. Knecht, H. Neufeld, H. Rupertsberger and P. Talavera, Radiative corrections to K(l3) decays, Eur. Phys. J. C 23 (2002) 121 [hep-ph/0110153] [INSPIRE].

[77] LHCf collaboration, Measurements of longitudinal and transverse momentum distributions for neutral pions in the forward-rapidity region with the LHCf detector, Phys. Rev. D 94 (2016) 032007 [arXiv: 1507.08764] [INSPIRE].

[78] TOTEM collaboration, Measurement of the forward charged particle pseudorapidity density in pp collisions at $\sqrt{s}=8 \mathrm{TeV}$ using a displaced interaction point, Eur. Phys. J. C 75 (2015) 126 [arXiv: 1411.4963] [INSPIRE].

[79] CMS collaboration, Measurement of the inclusive energy spectrum in the very forward direction in proton-proton collisions at $\sqrt{s}=13 \mathrm{TeV}$, JHEP 08 (2017) 046 [arXiv: 1701.08695] [INSPIRE].

[80] I. Esteban, M.C. Gonzalez-Garcia, A. Hernandez-Cabezudo, M. Maltoni and T. Schwetz, Global analysis of three-flavour neutrino oscillations: synergies and tensions in the determination of $\theta_{23}, \delta_{C P}$ and the mass ordering, JHEP 01 (2019) 106 [arXiv:1811.05487] [INSPIRE].

[81] C. Giunti and T. Lasserre, eV-scale Sterile Neutrinos, Ann. Rev. Nucl. Part. Sci. 69 (2019) 163 [arXiv: 1901.08330] [INSPIRE].

[82] ICECube collaboration, IceCube Sterile Neutrino Searches, EPJ Web Conf. 207 (2019) 04005 [arXiv: 1902.06185] [INSPIRE].

[83] M. Blennow, E. Fernandez-Martinez, J. Gehrlein, J. Hernandez-Garcia and J. Salvado, IceCube bounds on sterile neutrinos above $10 \mathrm{eV}$, Eur. Phys. J. C 78 (2018) 807 [arXiv: 1803.02362] [INSPIRE].

[84] T. Ohlsson and H. Snellman, Three flavor neutrino oscillations in matter, J. Math. Phys. 41 (2000) 2768 [Erratum ibid. 42 (2001) 2345] [hep-ph/9910546] [INSPIRE].

[85] W. Li, J. Ling, F. Xu and B. Yue, Matter Effect of Light Sterile Neutrino: An Exact Analytical Approach, JHEP 10 (2018) 021 [arXiv:1808.03985] [INSPIRE].

[86] NOMAD collaboration, Final NOMAD results on $\nu_{\mu} \rightarrow \nu_{\tau}$ and $\nu_{e} \rightarrow \nu_{\tau}$ oscillations including a new search for $\nu_{\tau}$ appearance using hadronic $\tau$ decays, Nucl. Phys. B 611 (2001) 3 [hep-ex/0106102] [INSPIRE].

[87] NOMAD collaboration, Search for $\nu_{\mu} \rightarrow \nu_{e}$ oscillations in the NOMAD experiment, Phys. Lett. B 570 (2003) 19 [hep-ex/0306037] [INSPIRE].

[88] M. Dentler et al., Updated Global Analysis of Neutrino Oscillations in the Presence of eV-Scale Sterile Neutrinos, JHEP 08 (2018) 010 [arXiv:1803.10661] [INSPIRE].

[89] A.I. Belesev et al., An upper limit on additional neutrino mass eigenstate in 2 to $100 \mathrm{eV}$ region from 'Troitsk nu-mass' data, JETP Lett. 97 (2013) 67 [arXiv:1211.7193] [INSPIRE]. 
[90] A.I. Belesev et al., The search for an additional neutrino mass eigenstate in the 2-100 eV region from 'Troitsk nu-mass' data: a detailed analysis, J. Phys. G 41 (2014) 015001 [arXiv: 1307.5687] [INSPIRE].

[91] DsTau collaboration, DsTau: Study of tau neutrino production with $400 \mathrm{GeV}$ protons from the CERN-SPS, JHEP 01 (2020) 033 [arXiv: 1906.03487] [INSPIRE].

[92] A. Bacchetta, D. Boer, C. Pisano and P. Taels, Gluon TMDs and NRQCD matrix elements in $J / \psi$ production at an EIC, Eur. Phys. J. C 80 (2020) 72 [arXiv:1809.02056] [INSPIRE].

[93] U. D'Alesio, F. Murgia, C. Pisano and P. Taels, Azimuthal asymmetries in semi-inclusive $J / \psi+$ jet production at an EIC, Phys. Rev. D 100 (2019) 094016 [arXiv:1908.00446] [INSPIRE].

[94] A. Bhattacharya, R. Enberg, M.H. Reno, I. Sarcevic and A. Stasto, Perturbative charm production and the prompt atmospheric neutrino flux in light of RHIC and LHC, JHEP 06 (2015) 110 [arXiv: 1502.01076] [INSPIRE].

[95] R. Gauld, J. Rojo, L. Rottoli, S. Sarkar and J. Talbert, The prompt atmospheric neutrino flux in the light of LHCb, JHEP 02 (2016) 130 [arXiv:1511.06346] [INSPIRE].

[96] PROSA collaboration, Prompt neutrino fluxes in the atmosphere with PROSA parton distribution functions, JHEP 05 (2017) 004 [arXiv: 1611.03815] [INSPIRE].

[97] G. Barr, T.K. Gaisser and T. Stanev, Flux of Atmospheric Neutrinos, Phys. Rev. D 39 (1989) 3532 [INSPIRE].

[98] T. Gaisser, Cosmic rays and particle physics, Cambridge University Press (1990) [INSPIRE]. 